


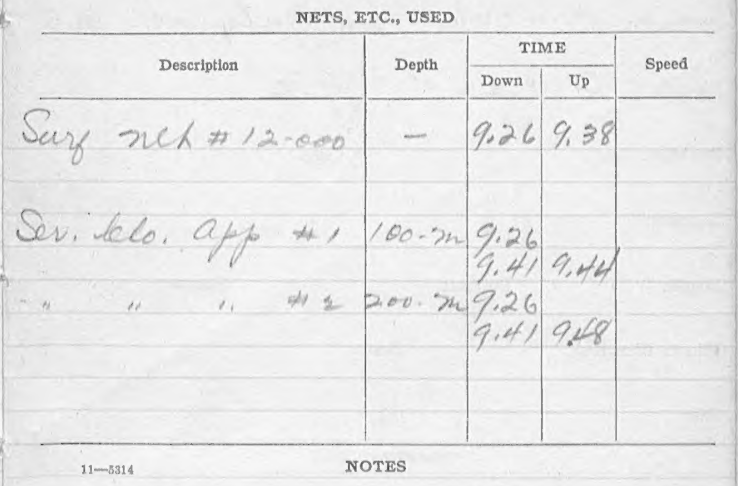

$$
\begin{aligned}
& 101 \\
& 29 \\
& 29
\end{aligned}
$$

masenger travel - 11/2 smi 
Station No. $\$ 6.6796$ Date: fully 281916 Hour: 958 Q.m.to 10.26 Q.m.

\section{Position: Lat.}

Long.

\section{Locality:}

\section{Depth:}

Bottom:

Sample:

Wind: Direction,

Sea:

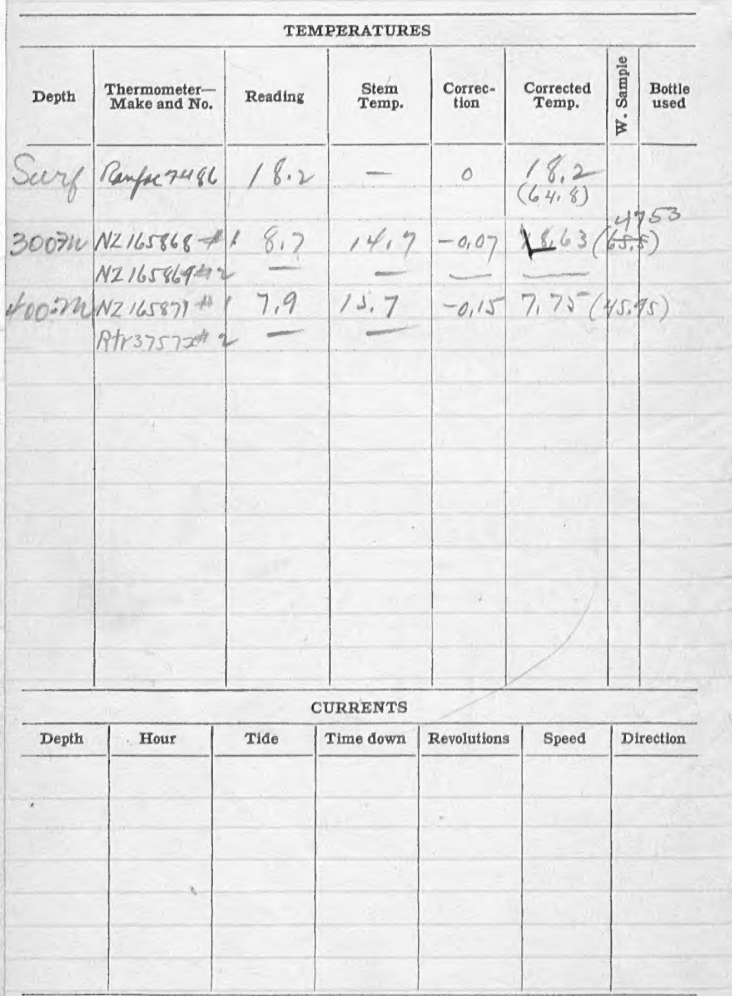

Color of water:

REMARKS:
Force,

Sky:

CURRENT

$11-5314$
Transparency: 


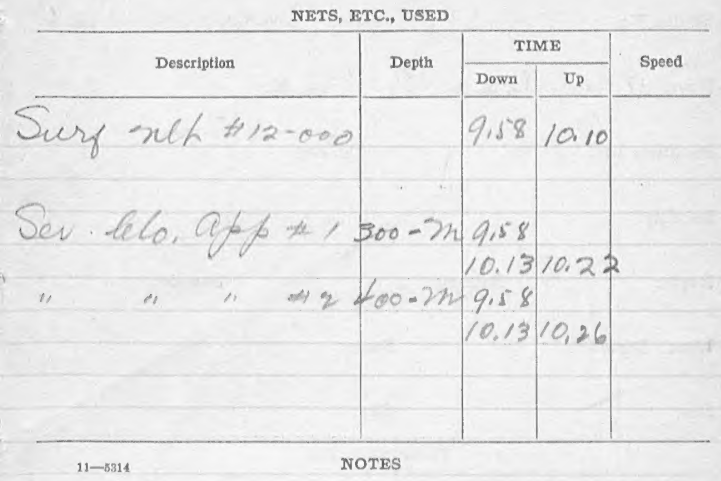

107 3nigh tomm- 458

$2 d$

$-10.13$

niger truvel 5- Miu

$$
\text { Psy } \left.\begin{array}{ccc}
W+5,2 \\
15,3
\end{array} \quad D / 6 .\right) \quad 86 \%
$$


Station No. It 6996 Date: Puly $26 \quad 1916$ Hour: 10.3 क Q.M. to 10,52 Q.m.

Position: Lat.

Long.

\section{Locality:}

Depth:

Bottom:

Sample:

f.

Wind: Direction,

Sea:
Sky:

Force,

TEMPERATURES

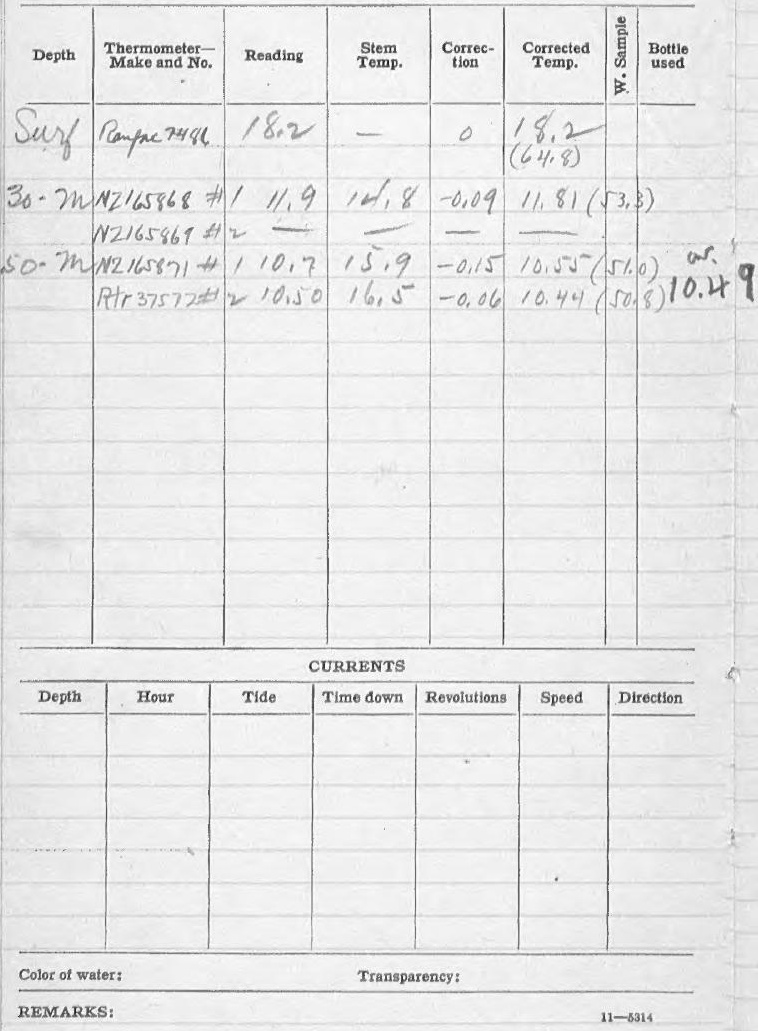




Station No. H 6797 Date: Quly 28 191
Hour: $12: 00 \quad$ M. to
Position: Lat. $32^{\circ}=40^{\prime}-00^{\prime \prime} \mathrm{M}$ Long. $118-02^{\circ}-42^{\prime \prime}$

\section{Locality:}

Depth:

Bottom:

Sample:

\section{Wind: Direction,}

Force,

Sea:

Sky:

TEMPERATURES

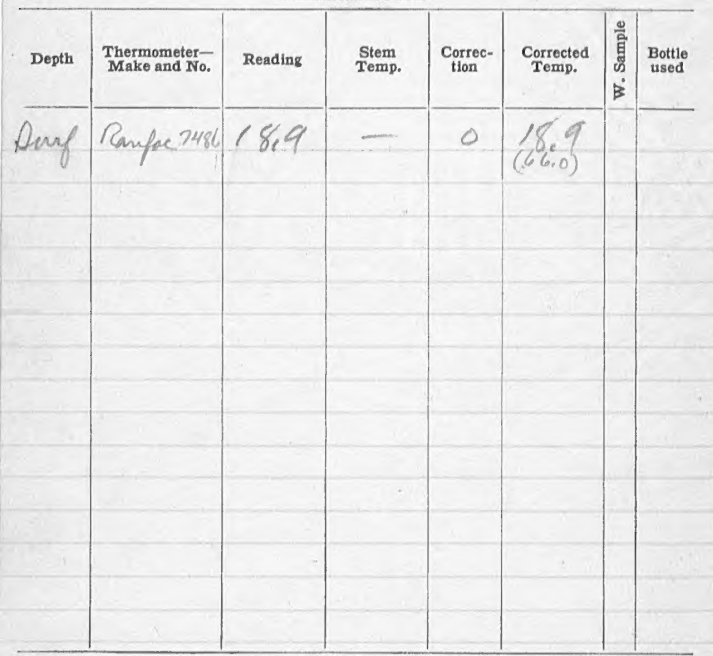

CURRENTS

\begin{tabular}{l|c|c|c|c|c|c}
\hline Depth & Hour & Tide & Time down & Revolutions & Speed & Directlon \\
\hline & & & & & \\
\hline & & & & & & \\
& & & & & & \\
& & & & & & \\
\hline
\end{tabular}

Transparency:

REMARKS: Uudu niay

$11-6314$ 
NETS, ETC., USED

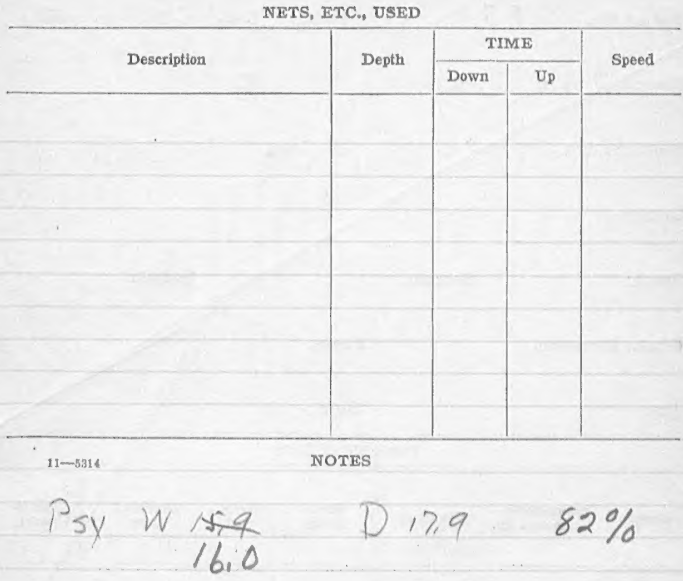

chait 5002 C.9 H.d. 
Station No. 6798

Date: Guby 28

1916

Hour: $17: 30$ P.M. to . $\mathrm{M}$.

Position: Lat. $32^{\circ}-40^{\prime}-12^{\prime \prime} \geqslant$ Long. $117-59^{\prime}-54^{\prime \prime}$

\section{Locality:}

Depth:

Bottom:

Sample:

Wind: Direction,

Force,

Sea:

Sky:

TEMPERATURES

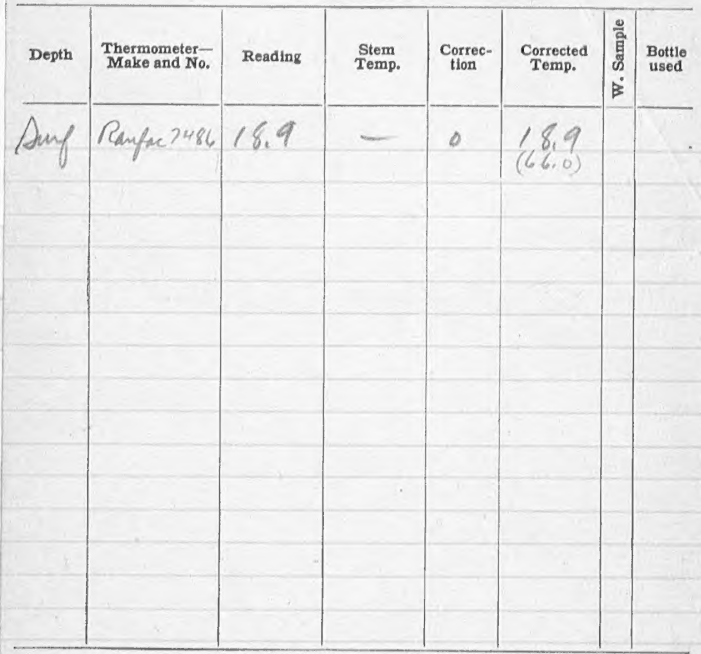

CURRENTS

\begin{tabular}{|c|c|c|c|c|c|c|}
\hline Depth & Hour & Tide & Time down & Revolutions & Speed & Direction \\
\hline & & & & & & \\
\hline & & & & & & \\
\hline & & & & & & \\
\hline & & & & & & \\
\hline & & & & & & \\
\hline Color of $w$ & \multicolumn{6}{|c|}{ Transparency: } \\
\hline REMARI & & & & & & $11-5314$ \\
\hline
\end{tabular}




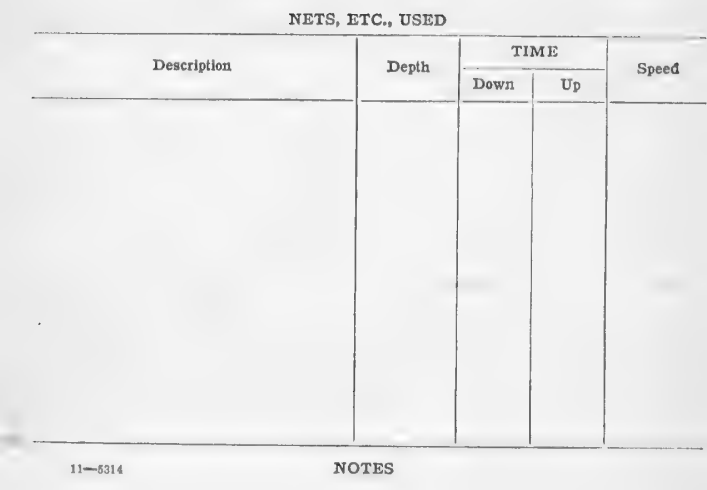

Chat 5002 E: 
Hour: 1 io $P$.M. to . M.

Position: Lat. $32^{\circ}-40^{\prime}-30^{\prime \prime}$ in Long. $117-5,-3.9 .11$

\section{Locality:}

Depth:

Bottom:

Sample:

Wind: Direction,

Force,

Sea:

Sky:

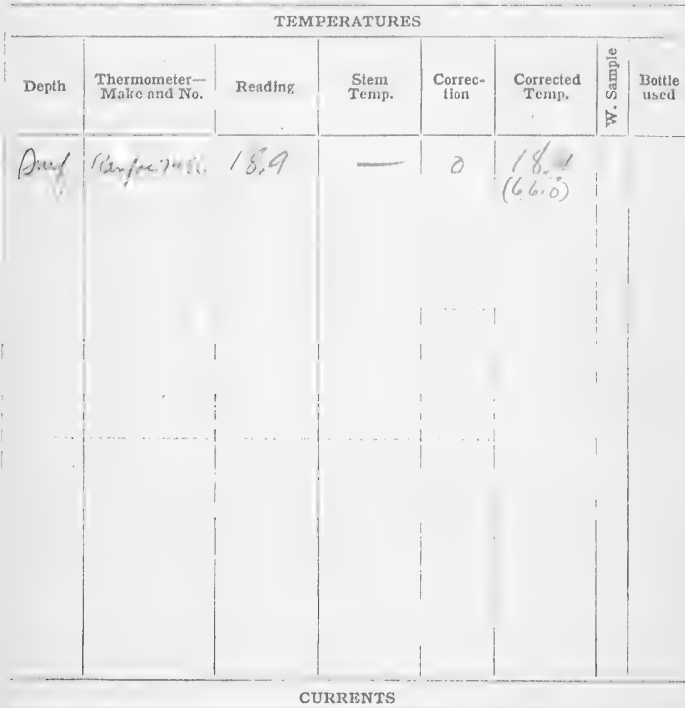

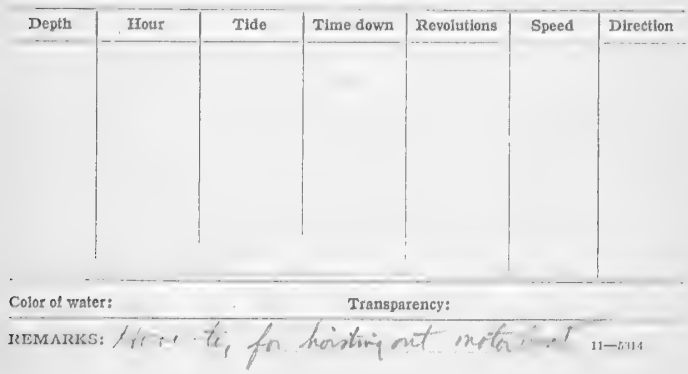


NETS, ETC., USED

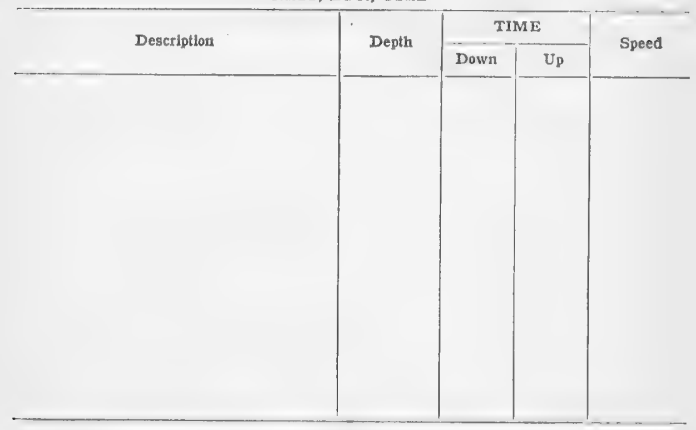

$11-5314$

NOTES

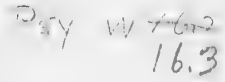

$-182=82 \%$

स. 
Station No. : \& $\because, 1$

Date: ()$i: ; \vdots$

191 ?

Hour: $/ 30 \quad P . M$. to $30 . . . . . . .$.

Position: Lat. 32-40-5/t of Long. $117-53,2 \%:$

Tocality:

Depth:

Bottom:

Sample:

Wind: Direction,

Force,

Sea:

Sky:

TEMPERATURES

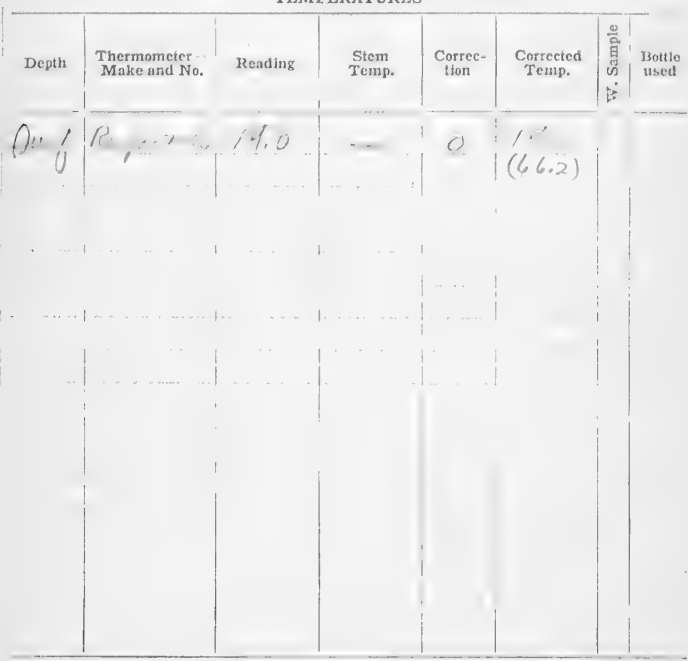

CURRENTS

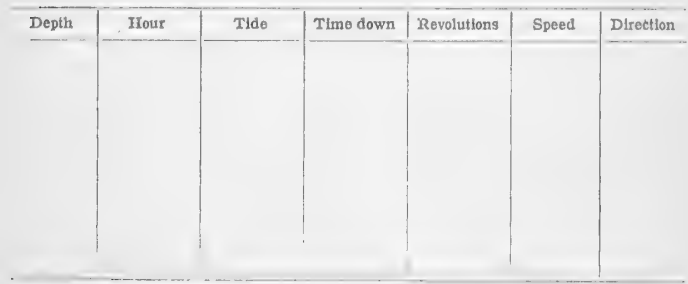

Transparency:

REMARKS: Lundu may

$11-8314$ 


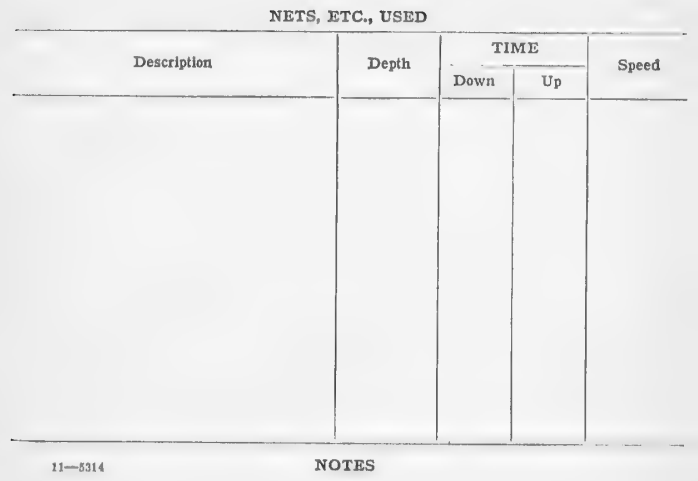

Chand 5002-C.8 i.S. 
Station No. / /

Date: Quly, :

$191 /$

Hour: 2 io 0 P.M. to - .M.

Position: Lat. $32-41-06^{\prime \prime}$ थ Long. if $7-50-30$ Locality:

Depth:

Bottom:

Sample:

Wind: Direction,

Force,

Sea:

Sky:

TEMRETATURES

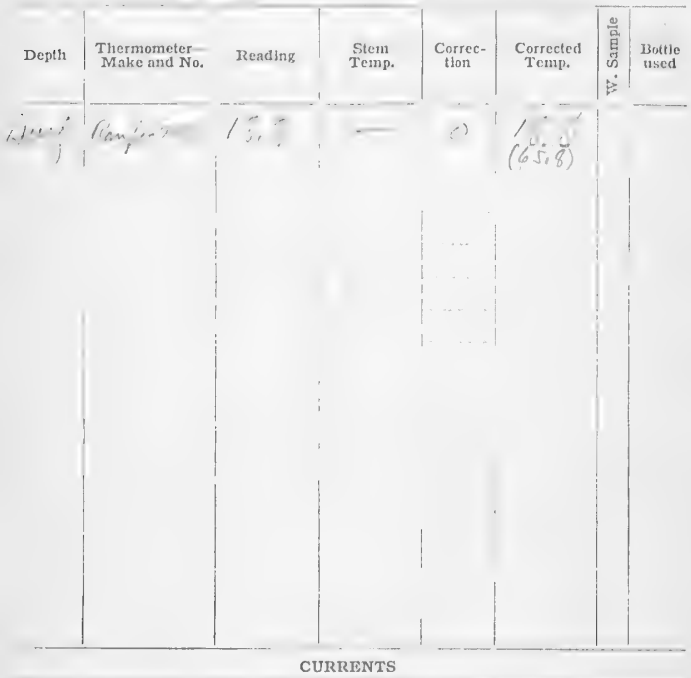

\begin{tabular}{l|l|l|l|l|l|l}
\hline Depth & Hour & Tide & Time down & Revoiutions & Speed & Direction \\
\hline & & & & & \\
& & & & & & \\
& & & & & & \\
& & & & & & \\
\end{tabular}

Color of water:

Transparency:

REMARKS:

Lholmen may

$11-8314$ 
NETS, ETC., USED

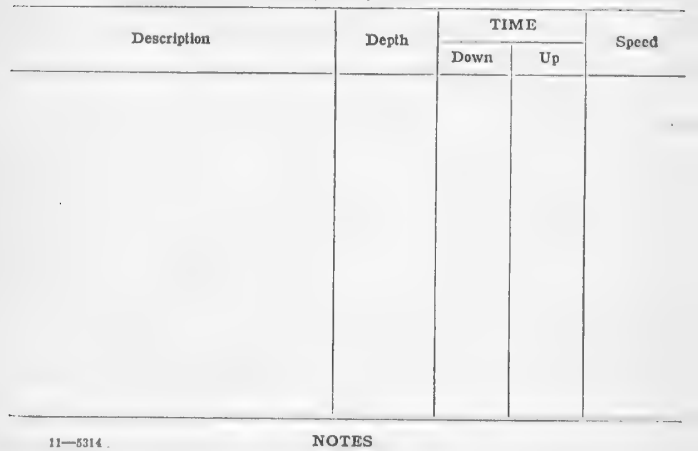

Psy $\frac{W+6.5}{16.6} D 18.5 \quad 82 \%$

chat $5002-$ C. I. S. 
Station Tro. $1 / \therefore \therefore$

Date: $(1,7,75$

$191 /$

Hour: $2: 30 \quad P . M$. to -

. M.

Position: Lat. $32^{\circ}-41^{\prime}-36^{\prime \prime} \geqslant$ Long. $117-45{ }^{\circ}, r^{\prime \prime}$

Locality:

Depth:

Bottom:

Sample:

Wind: Direction,

Force,

Sea:

Sky:

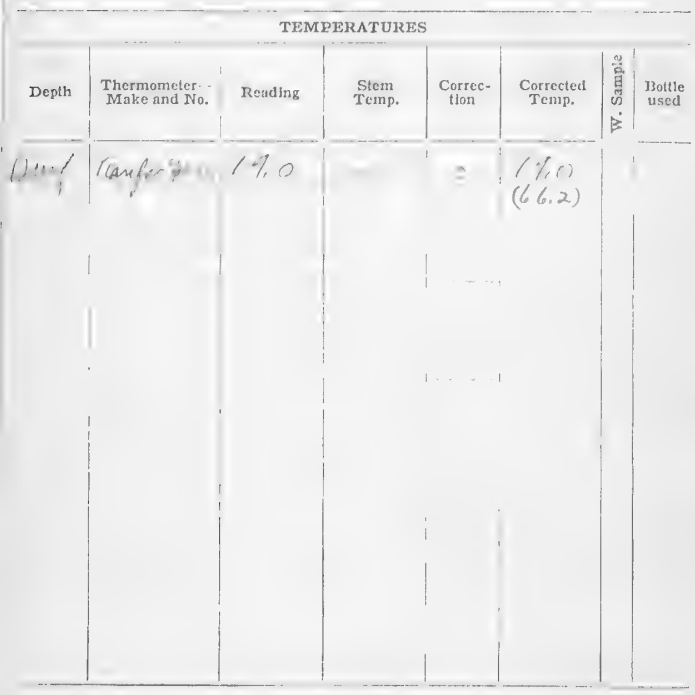

CURRENTS

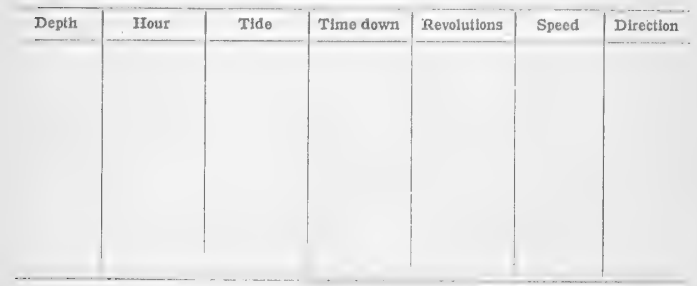

Color of water:

Transparency:

REMARKS:

thera rany

11- 1314 
NETS, ETC., USED

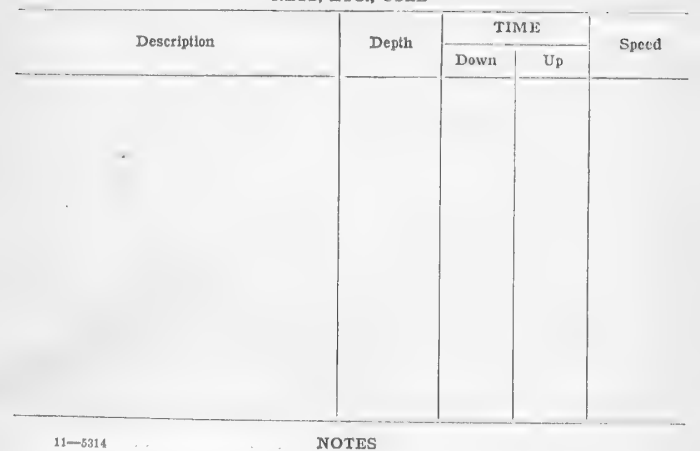

Chant 5e02 \&* * 
Station No. ;! ; ;,

Date: $\div i_{1 /} ;$

Hour: 3100 . M. to . M.

Position: Lat. $32^{\circ}-42^{\circ}-00^{\prime \prime}$ Long. : $7-3, \ldots=0$ is

\section{Locality:}

Depth:

Bottom:

Sample:

Wind: Direction,

Force,

Sea:

Sky:

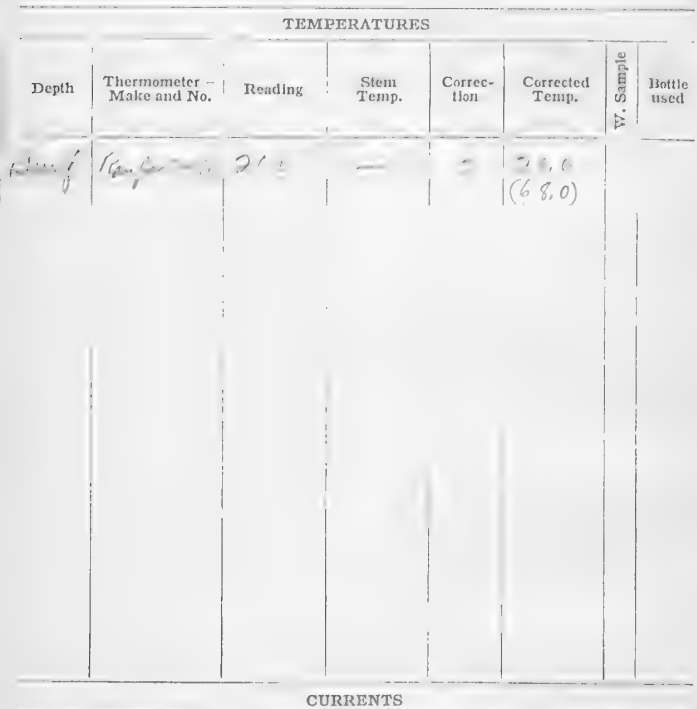

\begin{tabular}{l|l|l|l|l|l|l}
\hline Depth & Hour & Tide & Time down & Revolutions & Speed & Direction \\
\hline & & & & & & \\
\hline & & & & & & \\
\hline
\end{tabular}


NETS, ETC., USED

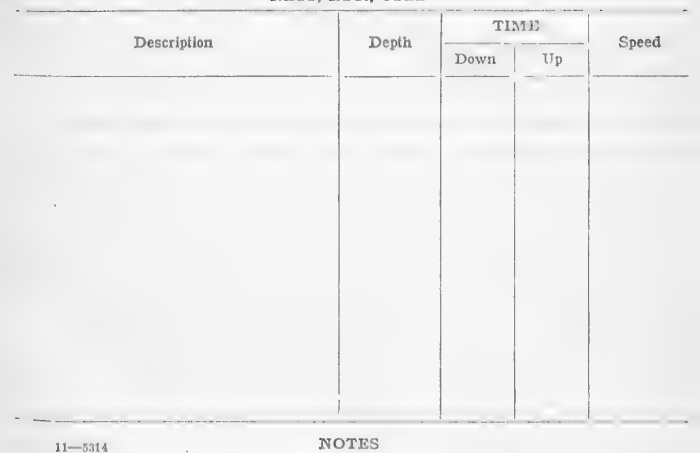

$$
11-5314
$$$$
\text { Notes }
$$

Psy $\begin{gathered}W+7.4 \\ 17.5\end{gathered} \quad D>0.0 \quad 78 \%$

Chang $500-4 \div 2 \%$ 
NETS, ETC., USED

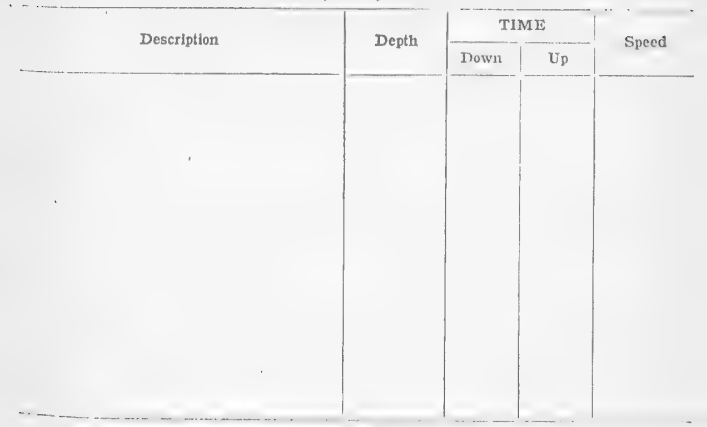


NETS, ITC., USED

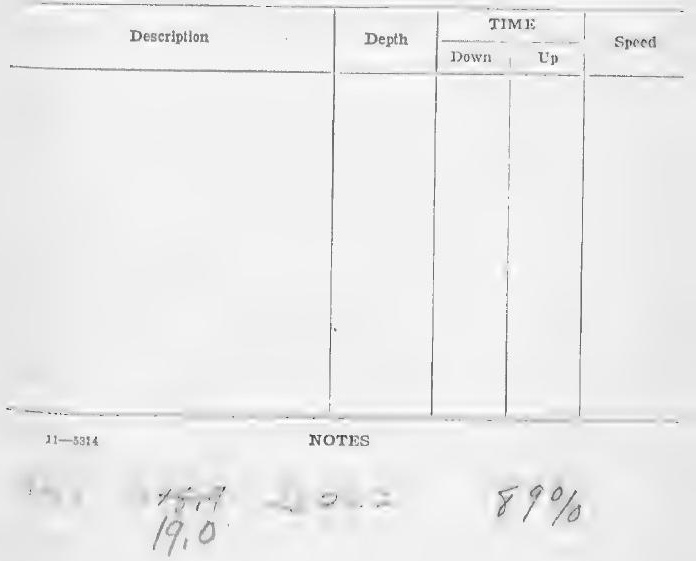

Chand 5002 - C.H. Y.S. 
Station No. 176806

Date: Orey 28

1916

How: " $/ 30$ P.M. to a

. M.

Position: Lat. $32^{\circ}-41^{\prime}-48^{\prime \prime}$ \& Long. $/ 17-23^{\prime}-30^{\prime \prime}$

Locality:

Depth:

Bottom:

Sample:

Wind: Direction,

Force,

Sea:

Sky:

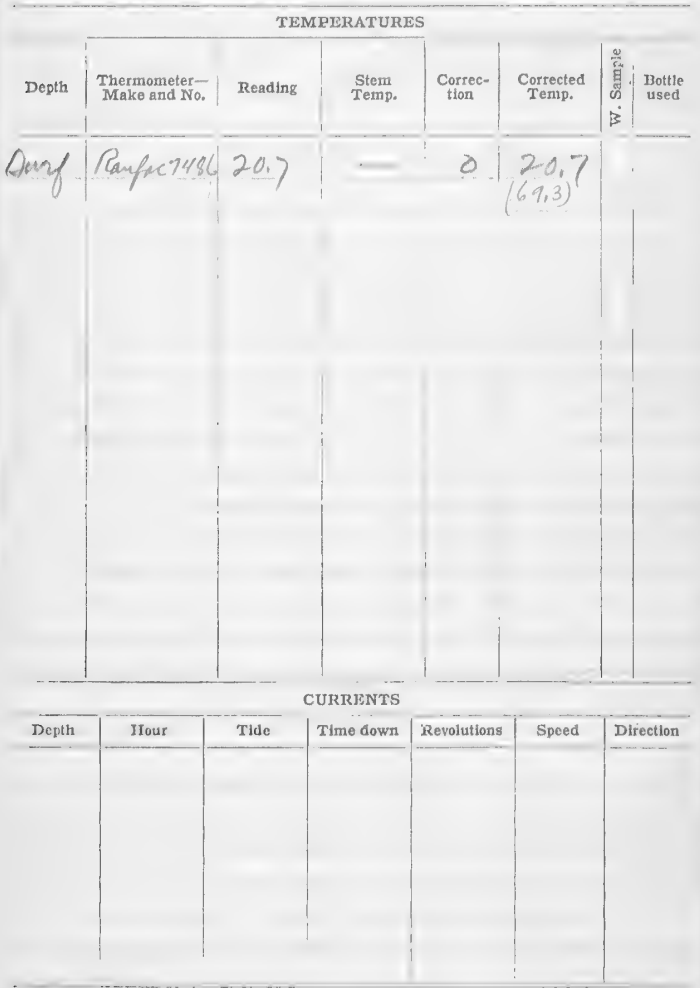

Transparency:

rEMARKS: Lluder voreq

$11-b 314$ 


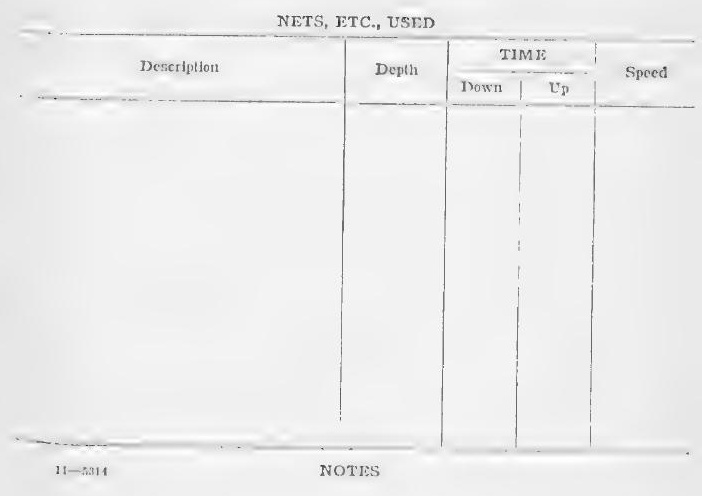

Chect roos- et a 
Station No. $+16 ; 0$ ?

Date: (fic) $>i^{\circ}$

Hour: $\sqrt{100} P$. M. to M.

Position: Lat. $32^{\circ}-40-30^{\prime \prime} \geqslant$ Long. $11^{\circ}-18 \div 37^{\circ}$

Locality:

Depth:

Bottom:

Sample:

Wind: Direction,

Force,

Sea:

Sky:

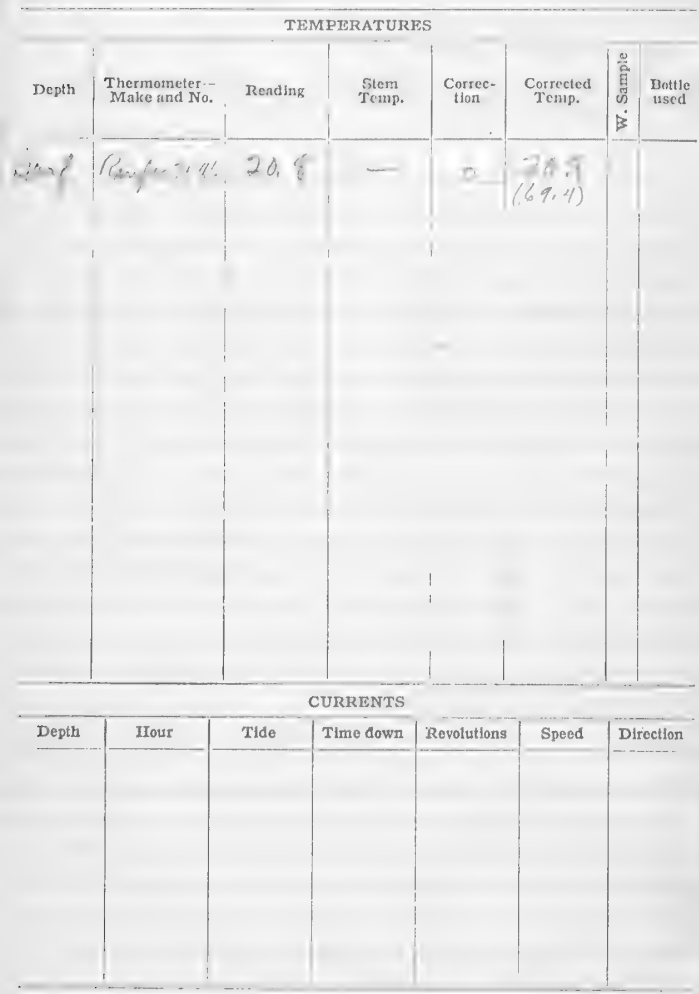

REMARKS: lenolu may 
NETS, ETC, USED

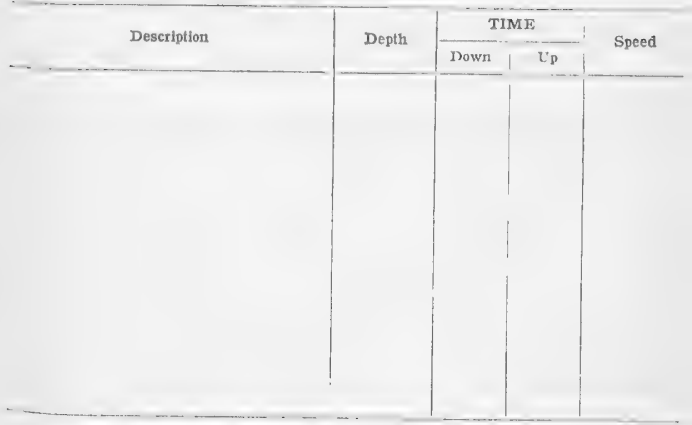

$11-5314$

Psy $w+7,0 \%$ ID 20.5

chant 5002 C.9 B.d.

(Ponit Loma light beare

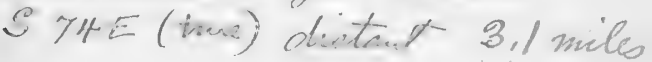


Station No. 146808

Date: 1916

Hour: 9ivo A.M. to -

. M.

Position: Lat. $32^{\circ}-40-22^{\prime \prime} \|$ Long. $117^{\circ}-13-55^{\prime \prime} W$

Locality:

Depth: Bottom: Sample:

Wind: Direction, Force,

Sea: Sky:
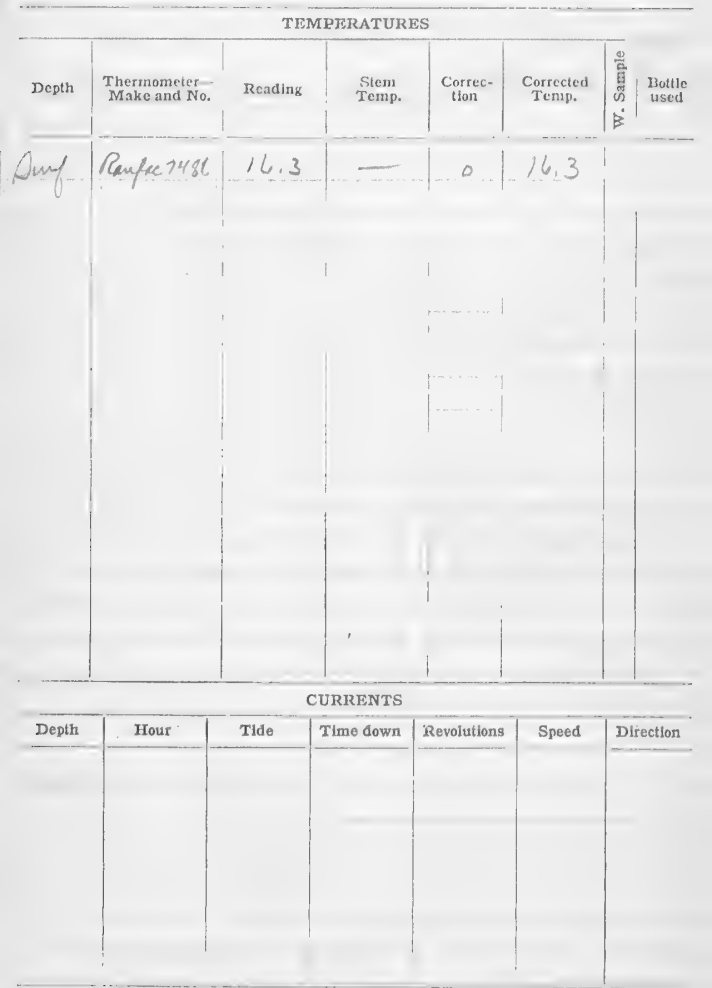

REMARKS: Liwher rmy 
NETS, ETC., USED

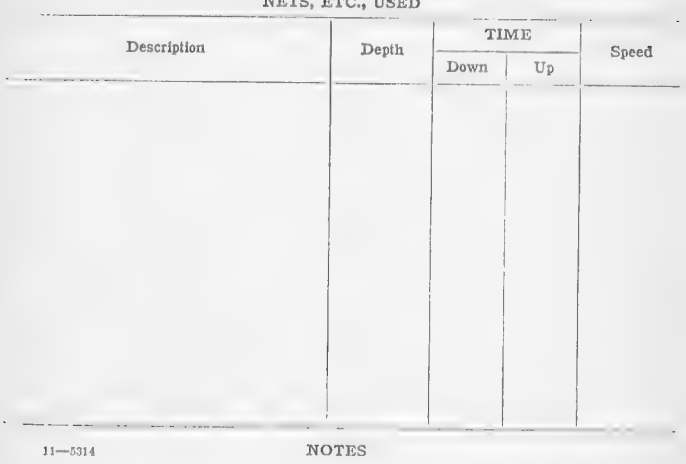

chait 1149 HO.

cicannel L'ight (Point Loma) beare is $152 / 3^{\circ}$ is, ofietant $3 / 4 \mathrm{mi}$. 
Hour: A:30 A.M. to -

. M.

Position: Lat. $32^{\circ}-36^{\prime}-33^{\prime \prime} \cup$ Long. $117^{\circ}-15^{\prime}-22^{\prime \prime} W$

Locality:

Depth:

Bottom:

Sample:

Wind: Direction,

Force,

Sea:

Sky:

Sea

TEMRERATURES

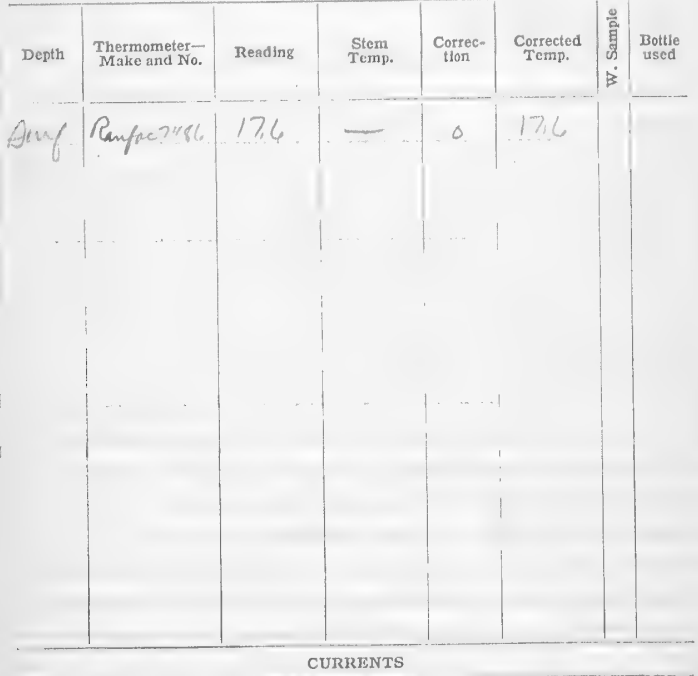

\begin{tabular}{l|l|l|l|l|l|l|}
\hline Depth & Tlour & Tide & Time down & Revolutions & Speed & Direction \\
\hline & & & & & \\
\hline & & & & & \\
& & & & & \\
& & & & &
\end{tabular}


NETS, ETC., USED

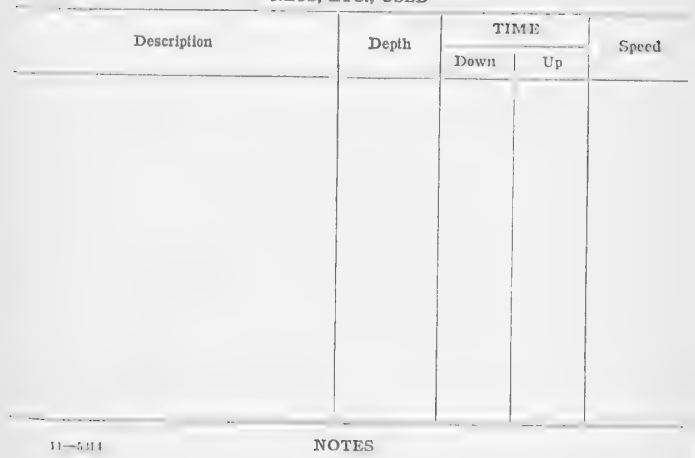

\footnotetext{
chait 1/49:00

Point Lom Lijht heare. $1110 \%{ }^{\circ} \mathrm{E}$ diatant 3.4 mole.
} 
Hour: $10: 00$ A.M. to . M.

Position: Lat. $32^{\circ}-32^{\prime}-48^{\prime \prime} \geqslant$ Long. $117-17-10^{\prime \prime}$

Locality:

Depth:

Bottom:

Sample:

Wind: Direction,

Force,

Sea:

Sky:

...

\begin{tabular}{|c|c|c|c|c|c|c|c|}
\hline Denth & $\begin{array}{l}\text { Thermometer- } \\
\text { Make ard No. }\end{array}$ & Reading & $\begin{array}{l}\text { Stem } \\
\text { Temp. }\end{array}$ & $\begin{array}{l}\text { Correc- } \\
\text { tion }\end{array}$ & $\begin{array}{l}\text { Corrected } \\
\text { Tcnilg. }\end{array}$ & 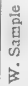 & $\begin{array}{l}\text { 13ittle } \\
\text { used }\end{array}$ \\
\hline
\end{tabular}

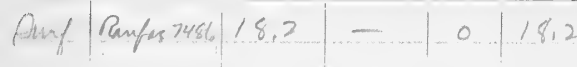

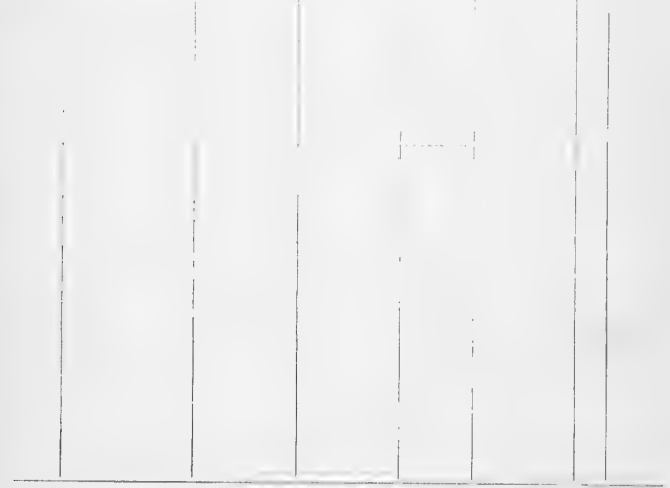

CURRIENS

\begin{tabular}{l|l|l|l|l|l|l}
\hline Depth & Tour & Tide & Time down & Revolutions & Speed & Direction \\
\hline & & & & & \\
\hline & & & & \\
& & & & &
\end{tabular}


NETS, IITC., USED

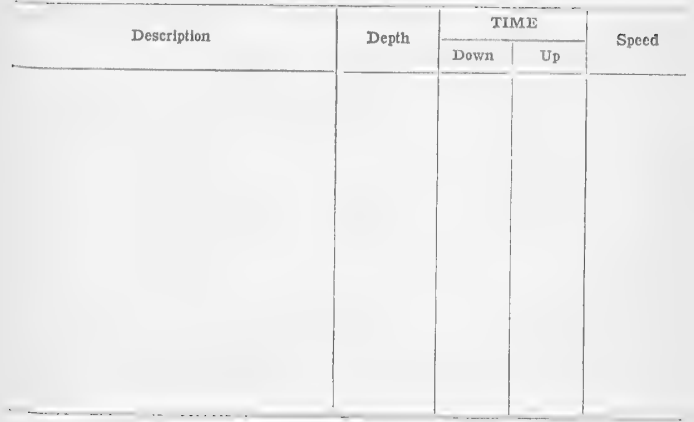

$11-5314$

\section{NOTIS}

$$
\begin{aligned}
& \text { PSV WVIH, DI, DI, }
\end{aligned}
$$

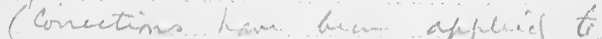

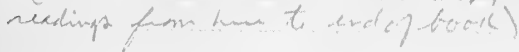

$$
\begin{aligned}
& \text { chait } 1149 \mathrm{HO} \text {. } \\
& \text { Point Joma light brans } \geqslant 163 / 1 \% \mathrm{E}
\end{aligned}
$$


NETS, ETC., USED

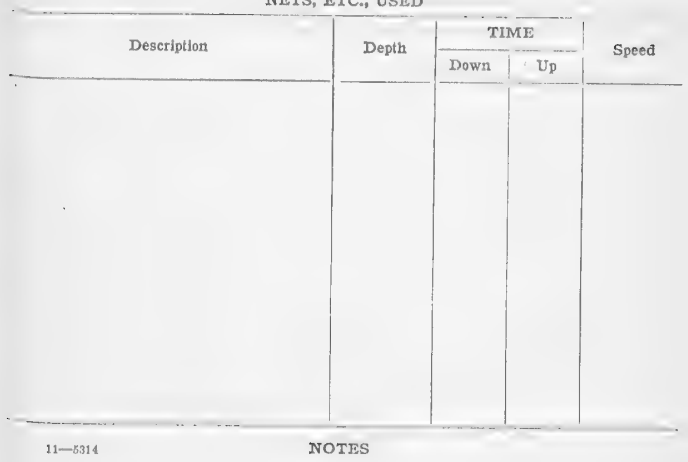

chait $1 / 49, \mathrm{H} . \mathrm{O}$.

Ponit Loma tight beare $1118^{2} / 3^{\circ} \mathrm{E}$ distant 10, it mobes. 
Station No. $H 6812$

Date:

Hour: $11: 00$ A. M. to . M.

Position: Lat. $32^{\circ}-22^{\prime}-00^{\prime \prime} \geqslant$ Long. $117-21^{\circ}-00^{\prime \prime}$ W

Locality:

Depth: Bottom: Sample:

Wind: Direction,

Force,

Sea:

Sky:

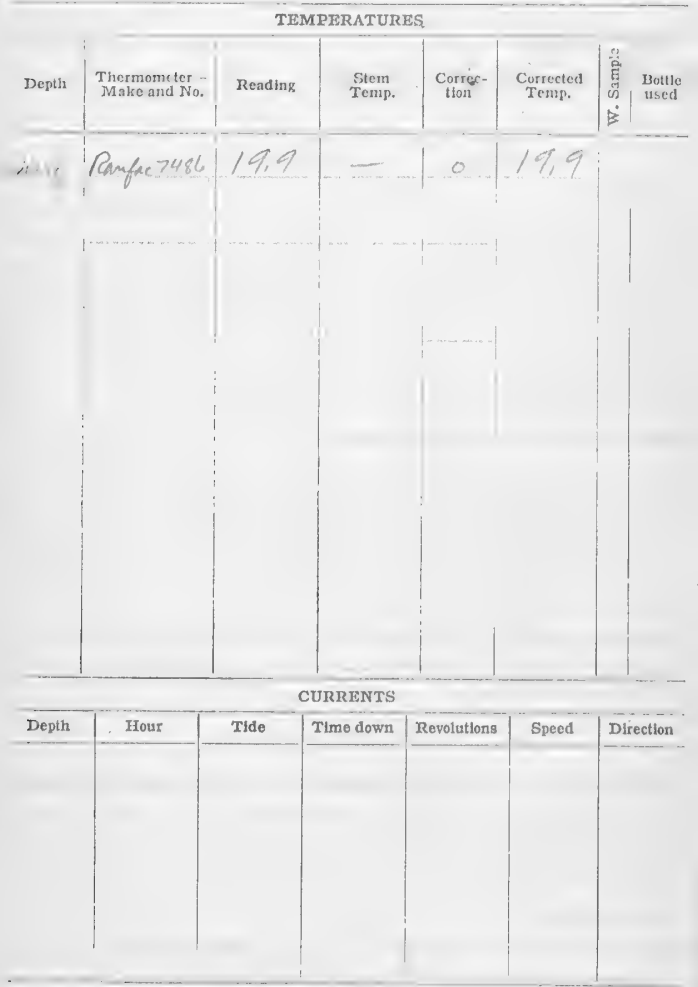


NETS, ITC., USED

Description

clemo kent 4.0 . 
NETS, ITC USED

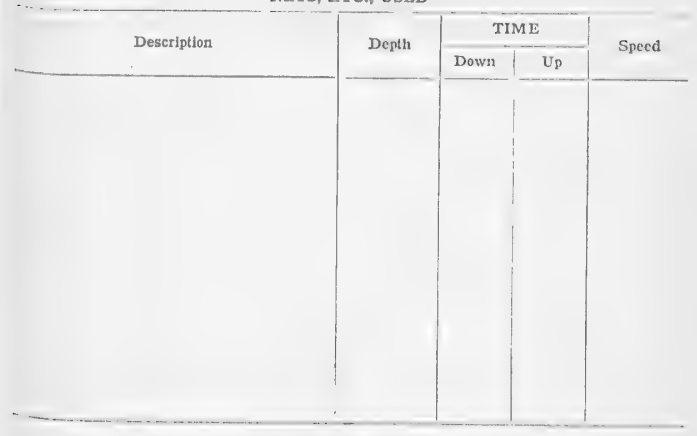

$11-5314$

NOTES

PSY. W 14.7 D 17,5

chait. 1006. H.O. 
Hour: $12: 30$ P.M. to - ...........

Position: Lat. $32^{\circ}-12^{\prime}-00^{\prime \prime} \geqslant$ Long. $117-23 \cdots 0 \ldots 1$

Locality:

Depth:

Bottom:

Sample:

Wind: Direction,

Force,

Sea:

Sky:

TEMPERATURES

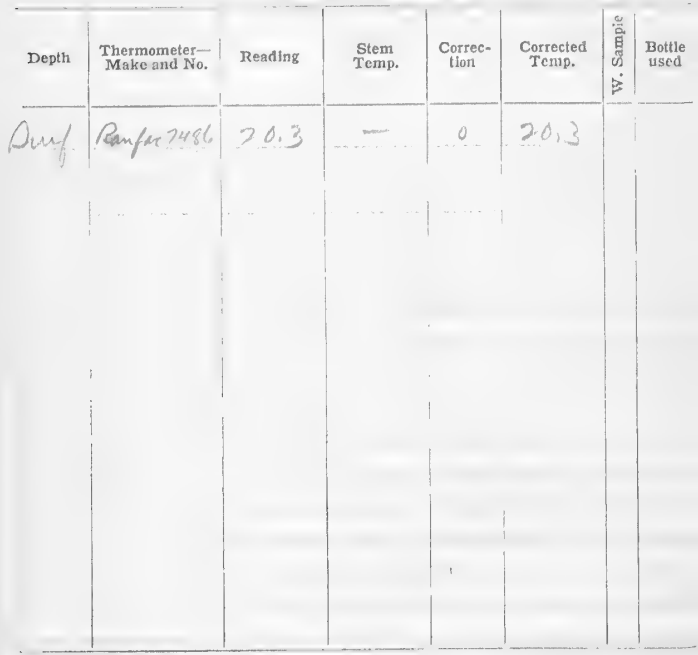

CURRENTS

\begin{tabular}{l|c|c|c|c|c|c|}
\hline Depth & IIour & Tide & Time down & Revolutions & Specd & Direction \\
\hline & & & & & \\
& & & & & \\
& & & & & & \\
\end{tabular}

Color of water:

Transparency:

REMARKS: Levele Irmy

$11-8314$ 
NETS, ETC., USED

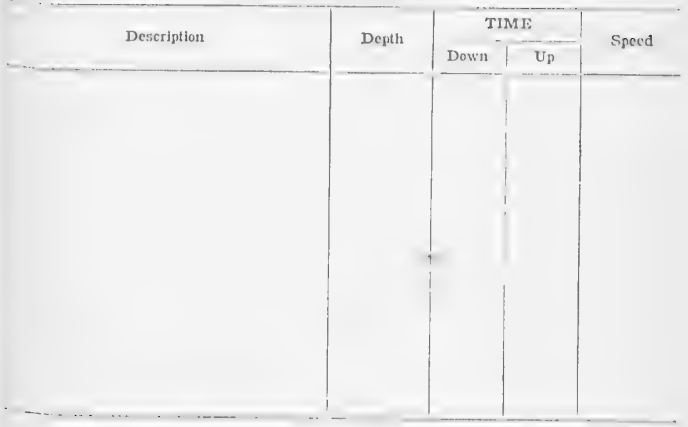


Hour: $/$ io $P$. M. to $=$

. M.

Position: Lat. $32^{\circ}-07-30^{\prime \prime} 20$ Long. $117-24-07^{\circ}$

\section{Locality:}

Depth:

Wind: Direction,

Sea:

Bottom:

Sample:

Force,

Sky:

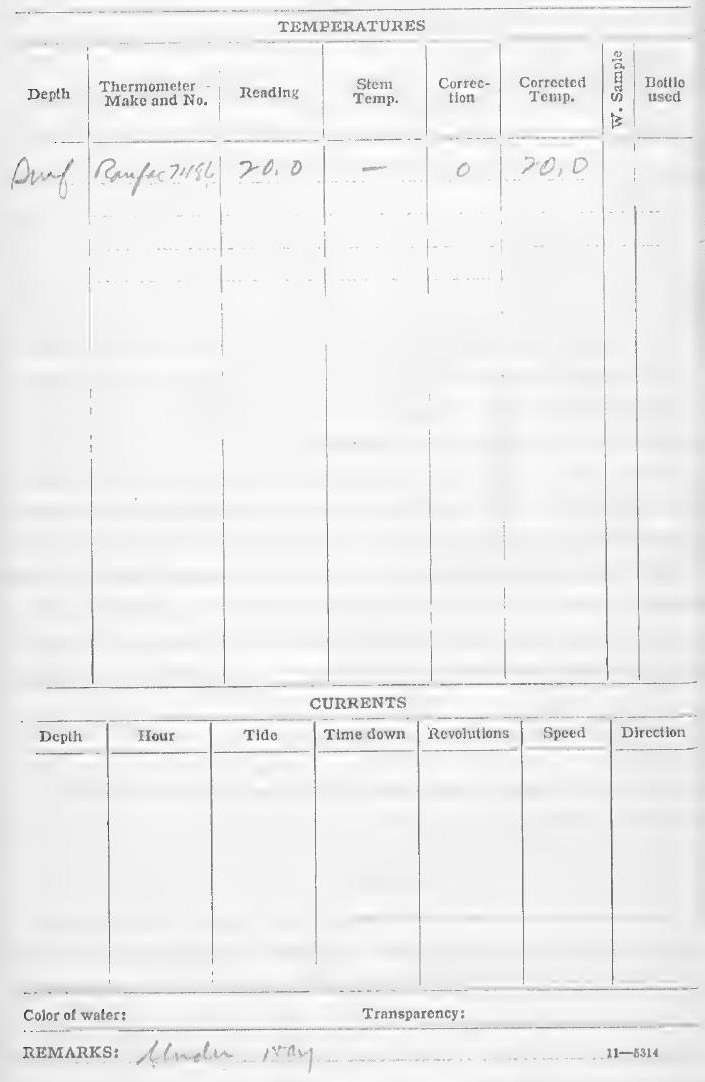


NETS, ETC., USED

Description

$11-5314$ NOTES

$1.15 .4,1 \cdots$

chet per no 


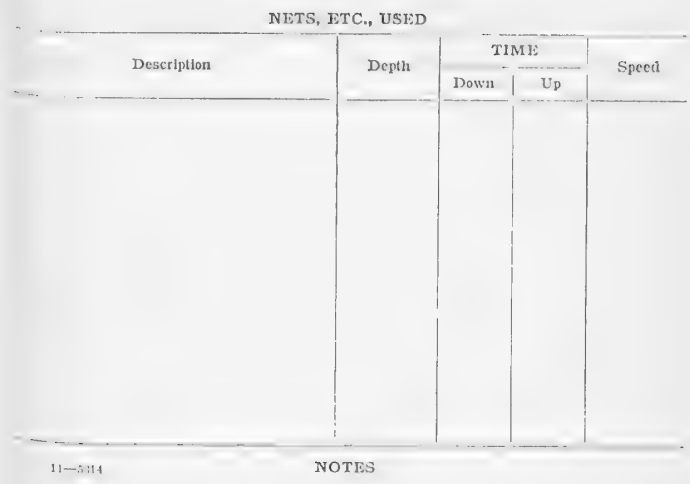

chat nan $-4=$ 
Station No. ' $;$,

Date: $>$ i : i

191.

Howr: $2: 00, M$. to $=. M$.

Position: Lat. $32^{\circ}-00^{\prime}-30^{\prime \prime} 27^{\text {Long. }} 117-2 y^{\prime}-1$

Locality:

Depth: Bottom: Sample:

Wind: Direction,

Force,

Sea:

Sky:

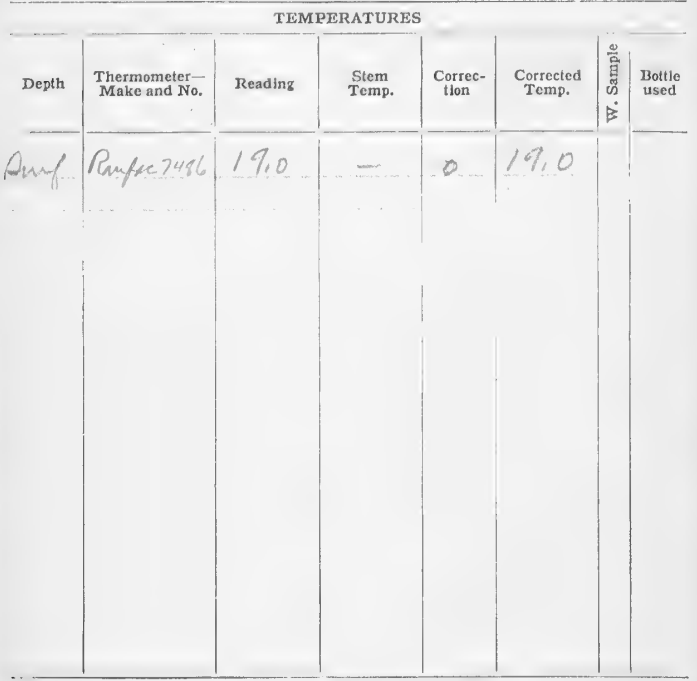

\begin{tabular}{|c|c|c|c|c|c|c|}
\hline \multicolumn{7}{|c|}{ CURRENTS } \\
\hline Depth & Hour & Tide & Time down & Revolutions & Speed & Direction \\
\hline & & & & & & \\
\hline & & & & & & \\
\hline & & & & & & \\
\hline & & & & & & \\
\hline & & & & $\sim \cdots$ & & \\
\hline & & & & & & \\
\hline & & & & & & \\
\hline
\end{tabular}

Transparency:

REMARKS: Cholum why 
NETS, ETC., USED

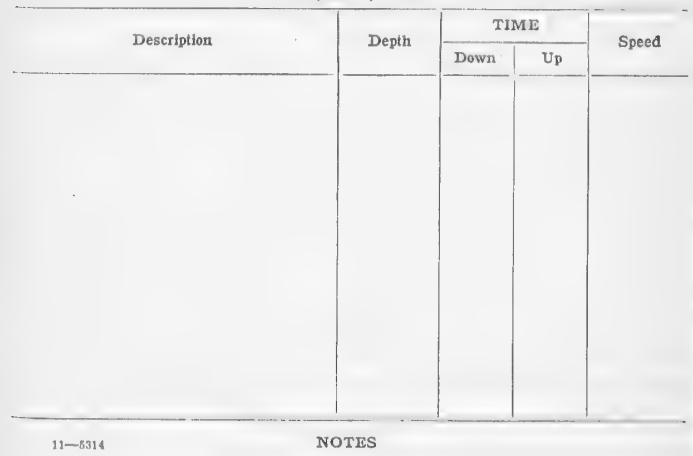

chact $1006-17.0$. 
Hour: $>130 P, M$, to $P$.

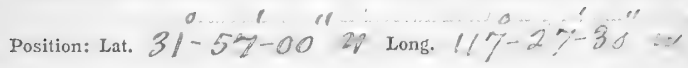
Locality:

Depth:

Bottom:

Sample:

Wind: Direction,

Sea:

TEMPTRATURES

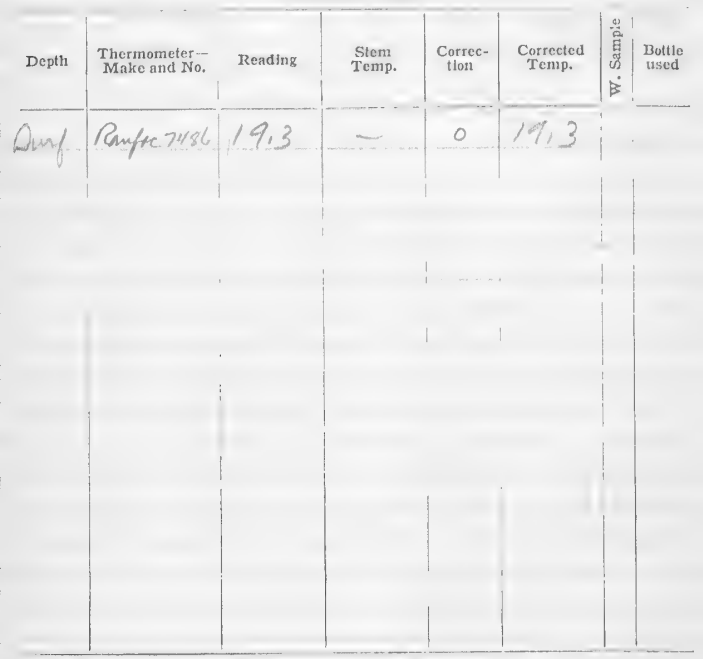

CURRENTS

\begin{tabular}{l|l|l|l|l|l|l|}
\hline Depth & Hour & Tide & Time down & Revolutions & Speed & Direction \\
\hline & & & & & \\
& & & & & \\
\end{tabular}

Color of water:

Transparency:

REMARKS: thath man

$11-5314$ 
NETS, ETC., USED

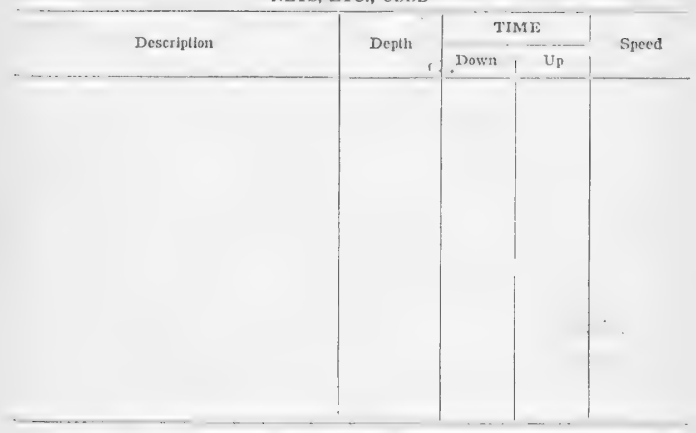


Station No. +16870

Date: Rug 17?

Hour: 3 io $0 \quad P$. M. to - .M.

Position: Lat. $3 i^{\circ}-53^{\prime}-00^{\prime \prime} \geq 1$ Long. $107^{\circ}-28^{\prime}-20^{\prime \prime}$

\section{Locality:}

Depth:

Bottom:

Sample:

Wind: Direction,

Sea:

TEMPERATURES

\begin{tabular}{|c|c|c|c|c|c|c|c|}
\hline Depth & $\begin{array}{l}\text { Thermometer-- } \\
\text { Make and No. }\end{array}$ & Reading & $\begin{array}{l}\text { Stem } \\
\text { Temp. }\end{array}$ & $\begin{array}{l}\text { Correc- } \\
\text { tion }\end{array}$ & $\begin{array}{l}\text { Corrected } \\
\text { Temp. }\end{array}$ & $\begin{array}{l}\text { 㡍 } \\
02 \\
3\end{array}$ & $\begin{array}{l}\text { Jottle } \\
\text { used }\end{array}$ \\
\hline
\end{tabular}

(s)

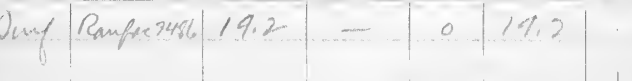

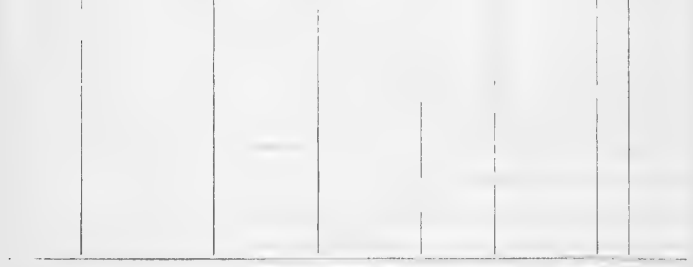

CURRENTS

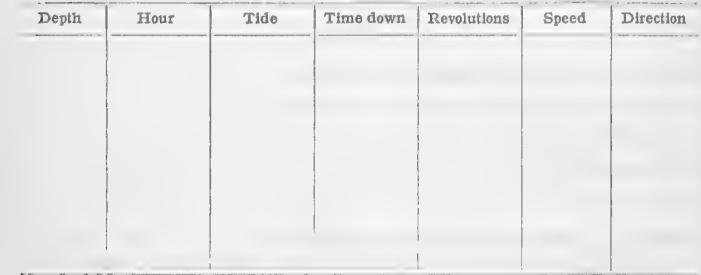

Color of water:

Transparency:

RIEMARKS: 
NETS, ITC., USED

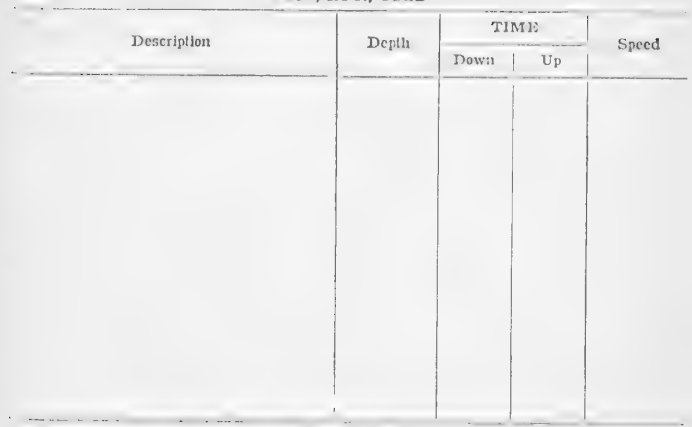

$21-531$ NOTES

$\because 1, \quad \therefore \quad 14,9 \div \cdots 1$

Cisentis is 
Hour: $3: 30$ P. M. to . M.

Position: Lat. $31-49-00^{\prime} P$ Long $117^{0}-29-00^{\prime}$

Locality:

Depth:

Bottom:

Sample:

Wind: Direction,

Force,

Sea:

Sisy:

TEMPERATURES

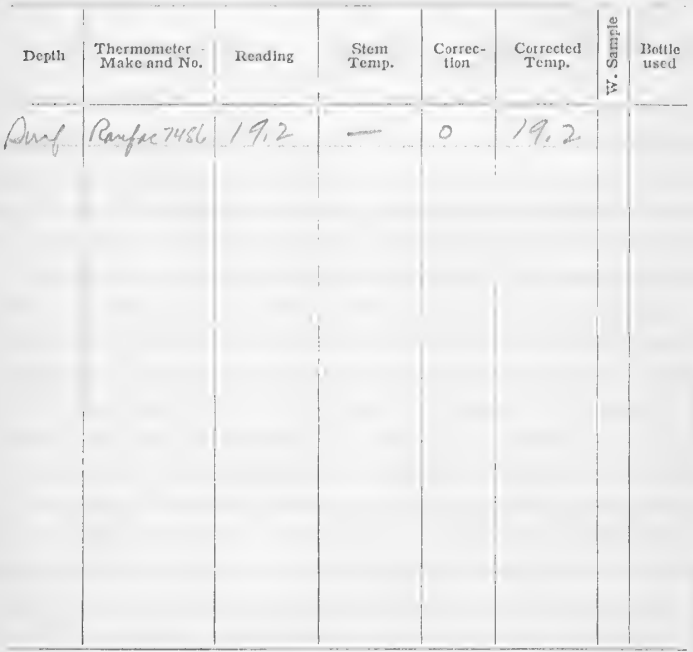

CURRENTS

\begin{tabular}{l|l|l|l|l|l|l}
\hline Deplh & Tour & Time & Time down & Revolutions & Speed & Directlon \\
\hline & & & & & \\
\hline
\end{tabular}


NETS, ITC., USED

Description

chant loo's ine. 
Station No, $/ \mathcal{H}$,

Date:

Hour: tio $P$. M. to m

. II.

Position: Lat. 31-45-00 21. Long. $11 \%-30^{\circ}-0 d^{\prime \prime}$

Locality:

Depth:

Bottom:

Sample:

Wind: Direction,

Force,

Sea:

Sky:

TEMPERATURFS

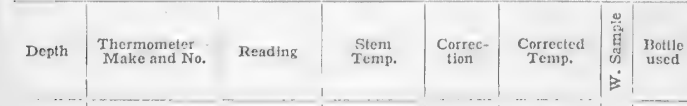

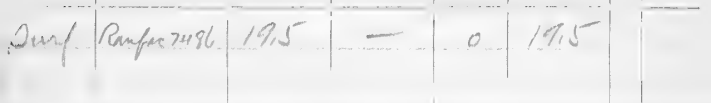

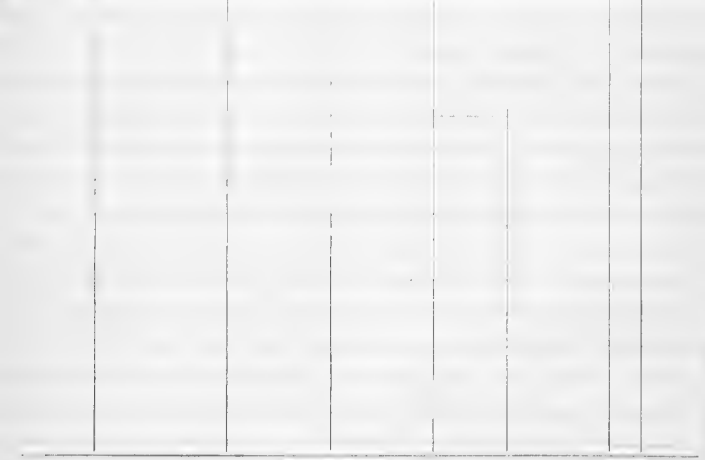

\begin{tabular}{|l|l|l|l|l|l} 
CURRENTS & Timo down & Revolutions & Speod & Direction \\
\hline Depth & Tide & & & & \\
\hline & & & & & \\
& & & & & \\
\end{tabular}


Hour: $4: 30$. M. to . M.

Position: Lat. $3 i^{0}-42-00^{\prime}$ थ Long. $117 \cdots 3: 37^{\prime}$,

\section{Locality:}

Depth:

Bottom:

Sample:

Wind: Direction,

Force,

Sea:

Sky:

TEMPERATURES

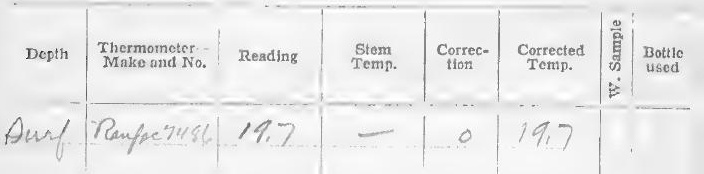

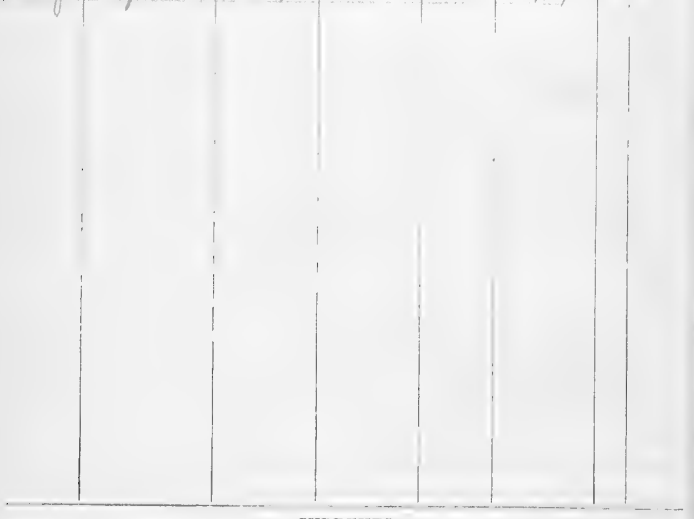

CURRENTS

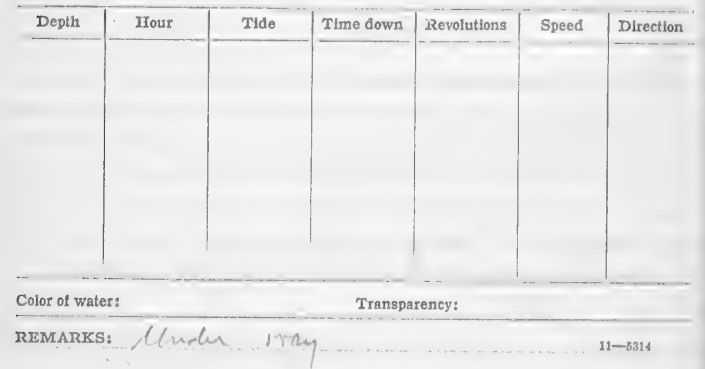


NETS, ETC., USED

Description

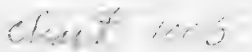


Hour: $5 i 00^{-}$M. to . M.

Position: Lat. 31-38-00 if Long. $117-3,2 \cdot 1$,

\section{Locality:}

Depth:

Bottom:

Sample:

Wind: Direction,

Force,

Sea:

Sky:

$$
\text { - }
$$

TEMPERATURES

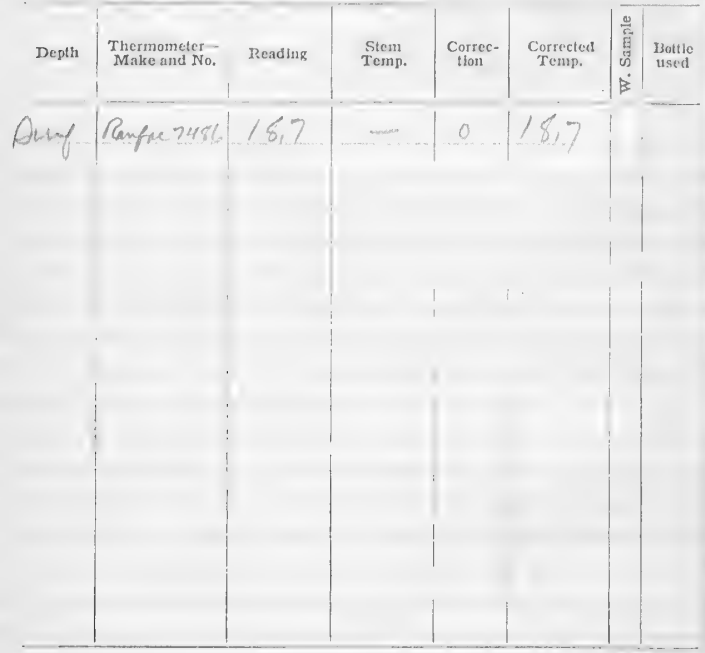

CURRENTS

\begin{tabular}{l|l|l|l|l|l|l}
\hline Depth & Hour & Tide & Timo down & Revolutions & Speed & Direction \\
\hline & & & & & \\
& & & & & \\
& & & & & \\
\end{tabular}

Transparency: 


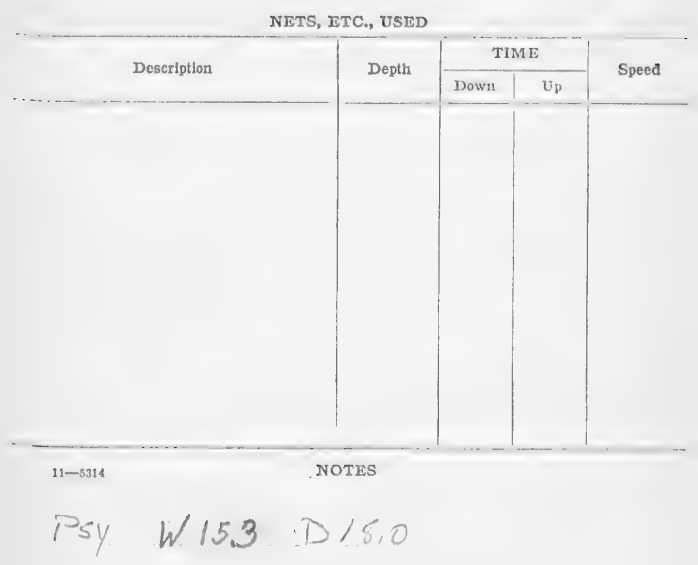

chaid $1006-H i O$ 
Station No. H $; \vdots$

Date:

$1 \cdots: 1$,

191 .

Hour: $\sqrt{13} \omega \sqrt{3}$. to - M.

Position: Lat. $31-34-00^{0}$ श Long. $117-33^{\prime}-00^{\prime \prime}$

Locality:

Depth:

Bottom:

Sample:

Wind: Direction,

Force,

Sea:

Slry:

TEMERRATURES

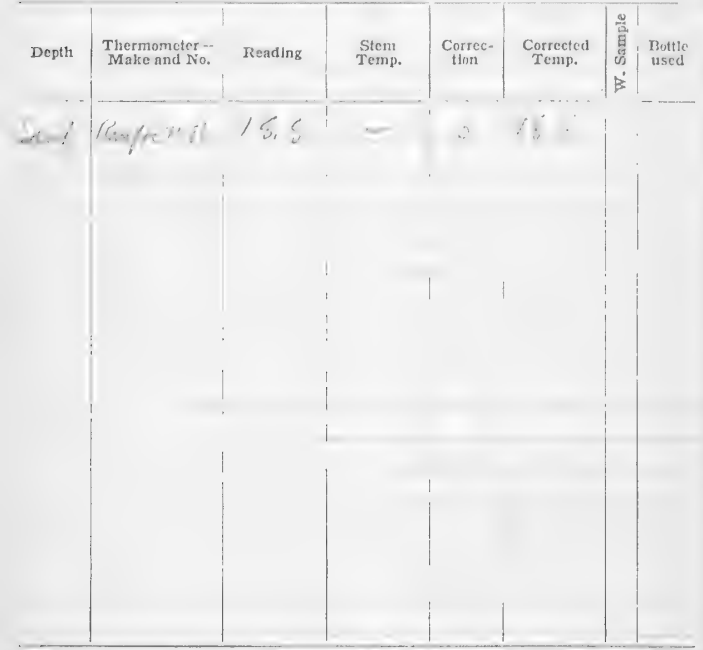

CURRENTS

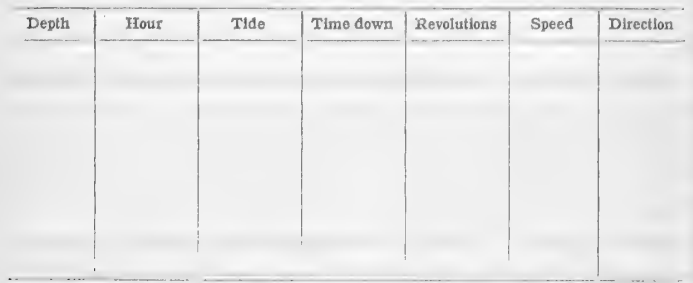

Color of water:

Transparency:

REMARKS: Lholu with $11-8314$ 
NITS, ETC., USED

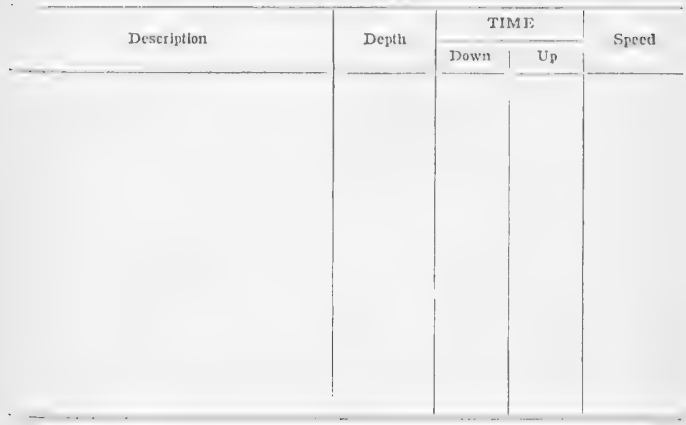


Station No. 46836

Hour: $6: 00$ M. to
Date: Cley 17

1916

. M.

Position: Lat. $31^{\circ}-30^{\prime}-00^{\prime \prime}$ if Long. $1 / \%-3 / \%-i$

Locality:

Depth:

Bottom:

Sample:

Wind: Direction,

Force,

Sea:

Siry:
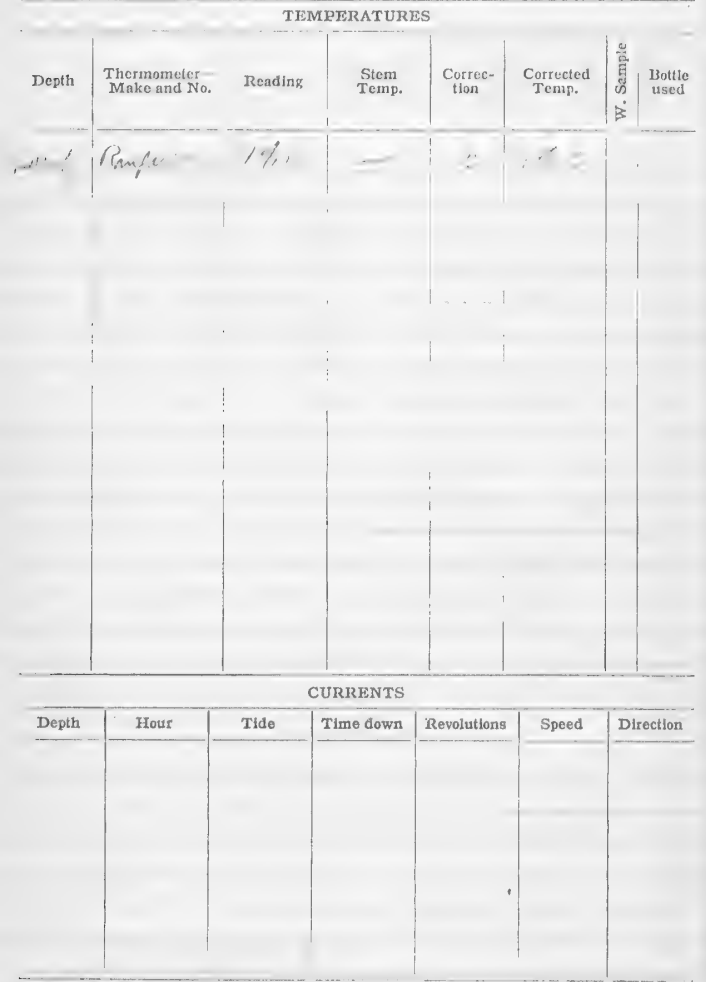

Color of water:

Transparency:

RTMARKS

Aude ivay 
NETS, ETC., USED

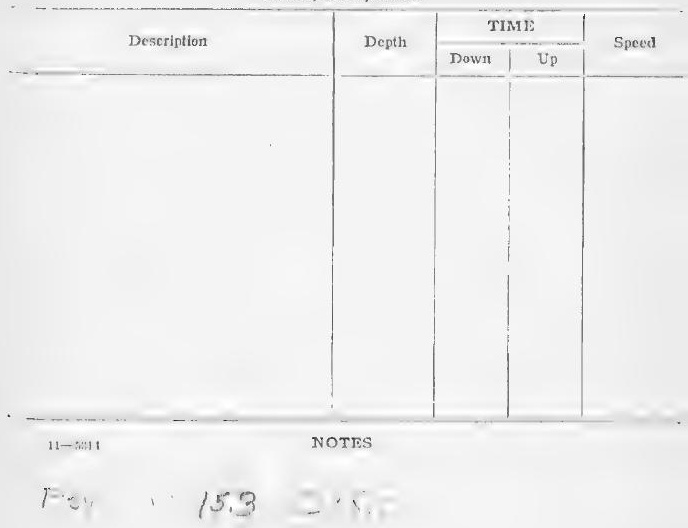


Hour: $6: 30 P$. M. to

Position: Lat. $31^{\circ}-27^{\prime}-00^{\prime \prime} \cap$ Long. $117^{\circ}-34^{\prime}-30^{\prime \prime}$ is. Locality:

Depth: $\quad$ Bottom: _... Sample:

Wind: Direction,

Sea:
Force,

Slky:

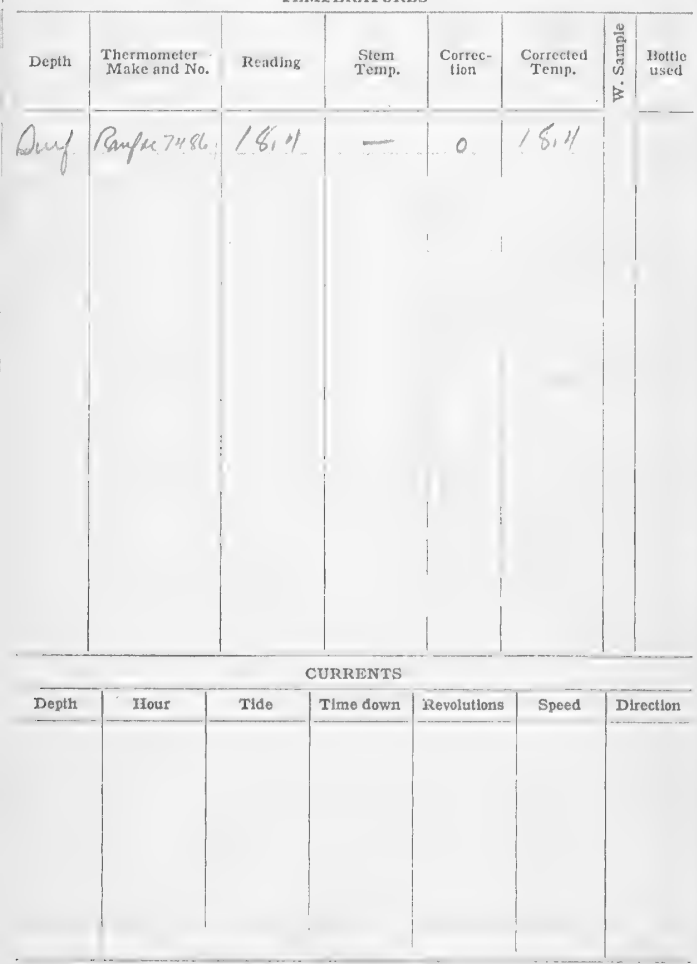


NETS, ETC., USED

Description

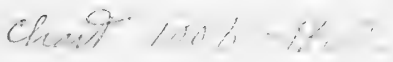


Hour: $7,0 D P . M$. to -

. M.

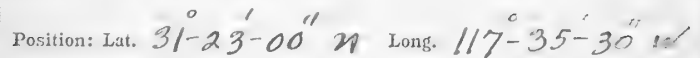

\section{Locality:}

\section{Depth:}

Bottom:

Sample:

Wind: Direction,

Sea:
Force,

Sky:

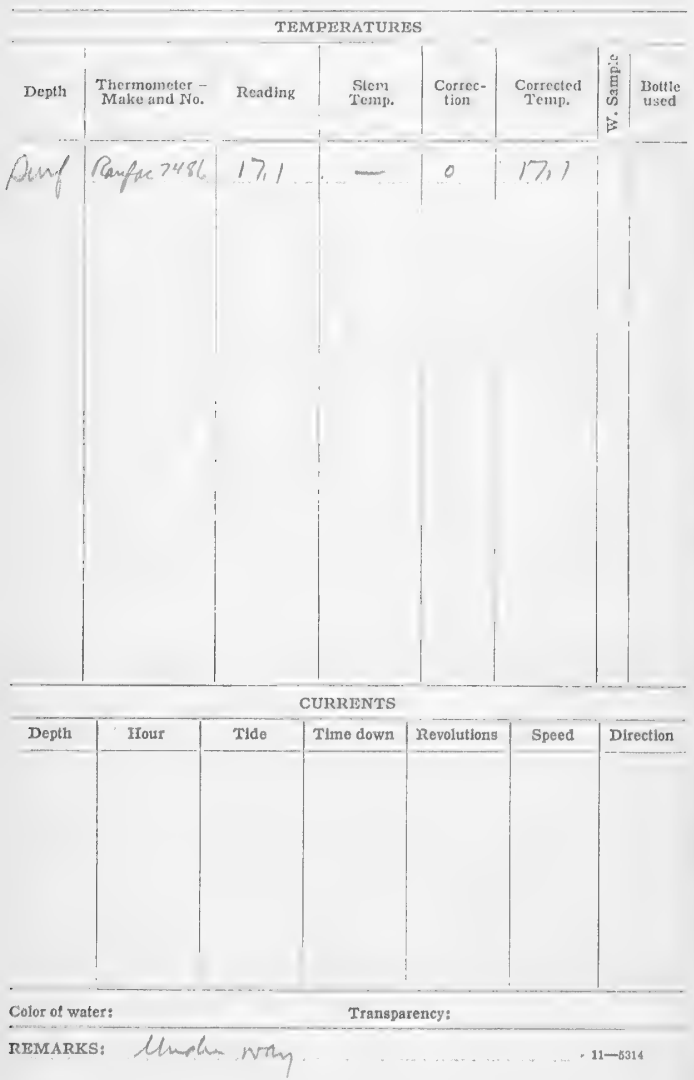




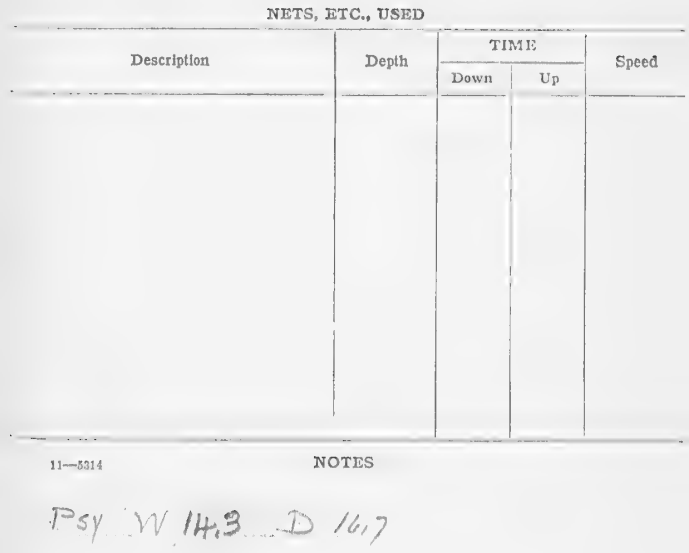

chat $1006-9.0$. 
Hour: $7: 30 P$ M. to $\ldots$ M.

Position: Lat. $3 f^{\circ}-17^{\prime}-00^{\prime \prime}$ in Long. $11 \%-3 i, 5 ;$, Locality:

Depth:

Wind: Direction,

Sea:

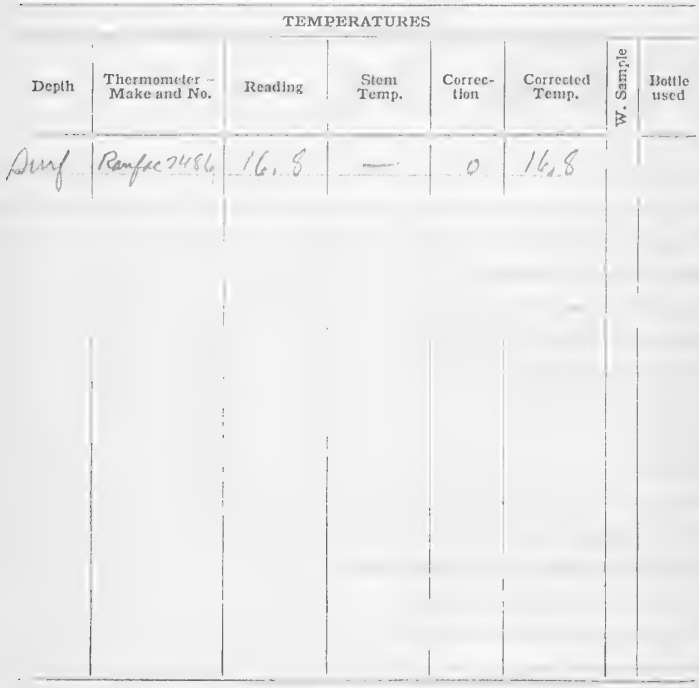

\section{CURRENTS}

\begin{tabular}{|c|c|c|c|c|c|c|}
\hline Depth & Hour & Tide & Time down & Revolutions & Speed & Direction \\
\hline & & & & & & \\
\hline & & & & & & \\
\hline & & & & & & \\
\hline & & & & & & \\
\hline & & & & & & \\
\hline
\end{tabular}




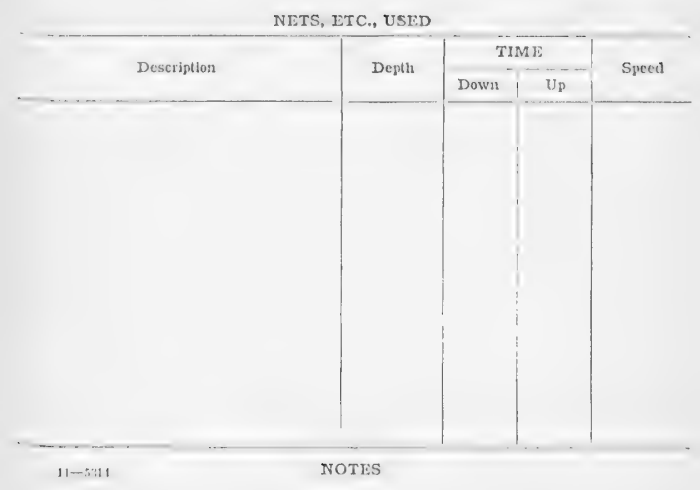

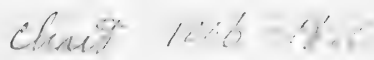


Hour: 8:00 $\mathrm{F}$.M. to . M.

Position: Lat. $31^{\circ}-16^{\prime}-00^{\prime \prime} 4$ Long. $117-37^{\circ}-00^{\prime \prime}$ w

Locality:

Depth:

Bottom:

Sample:

Wind: Direction,

Force,

Sea:

Sky:

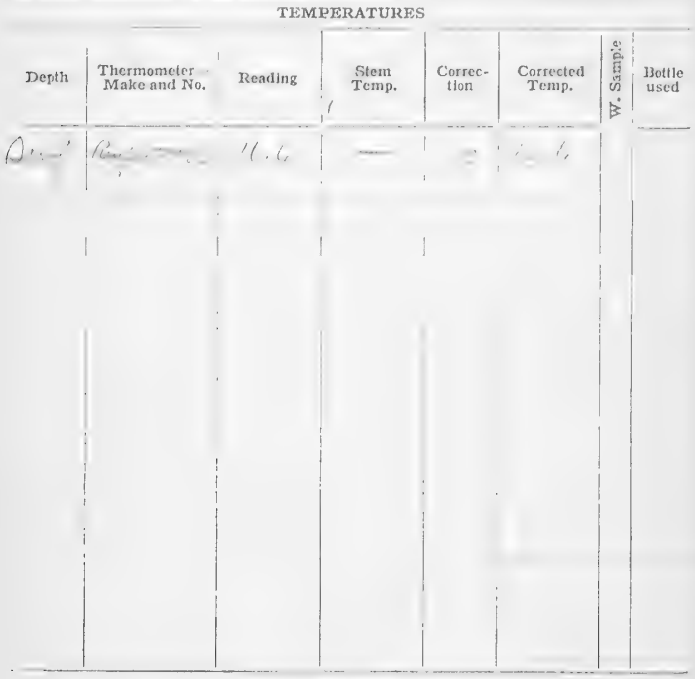

CURRRNTS

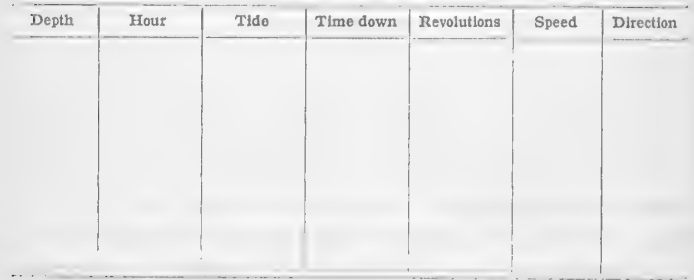


NETS, ETC., USED

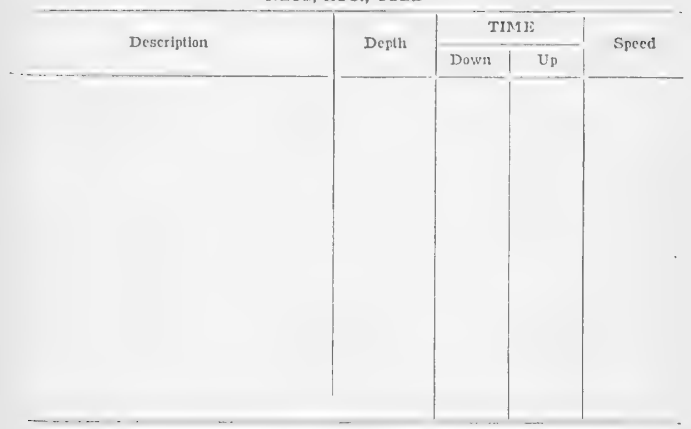

NOTES

$\cdots \quad 114.5 \quad \ldots \ldots$

chend tis: 
Hour: 8 i 30 P.M. to -

M.

Position: Lat. $31^{\circ}-12^{\prime}-00^{\prime \prime}$ \& Long. $117^{\circ}-38^{\prime} 00^{\prime \prime}$ al

Locality:

Depth:

Bottom:

Sample:

Wind: Direction,

Force,

Sea:

Slry:

TEMPERATURES

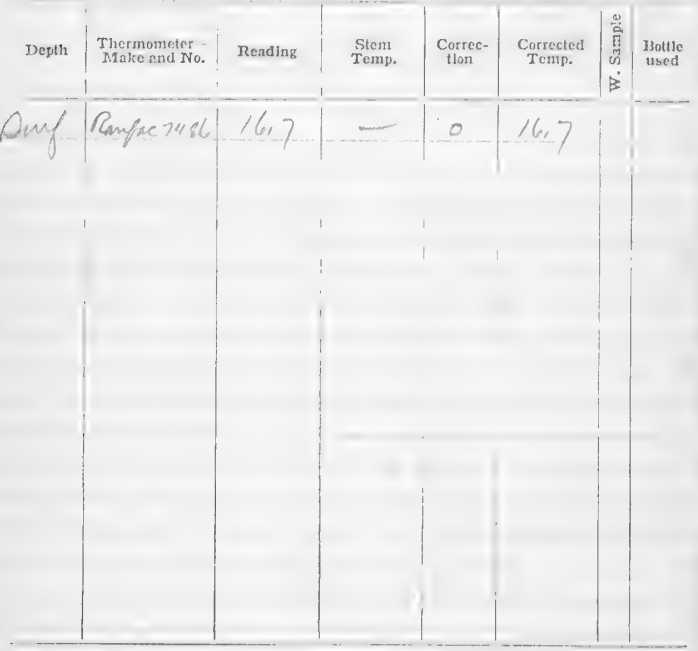

CURRENTS

\begin{tabular}{|c|c|c|c|c|c|c|}
\hline Depth & Hour & Tide & Time down & Revolutions & Speed & Direction \\
\hline & & & & & & \\
\hline & & & & & & \\
\hline & & & & & & \\
\hline & & & & & & \\
\hline & & & & & & \\
\hline
\end{tabular}


Hour: 7iOD P.M. to $P$.

Position: Lat. $31-08-00^{\circ} \mathrm{Q} / \mathrm{Long} 117^{\circ} 30^{\circ}$

Locality:

Depth:...$\ldots$. Bottom: ........... Sample:

Wind: Direction, Force,

Sea:

Sky:

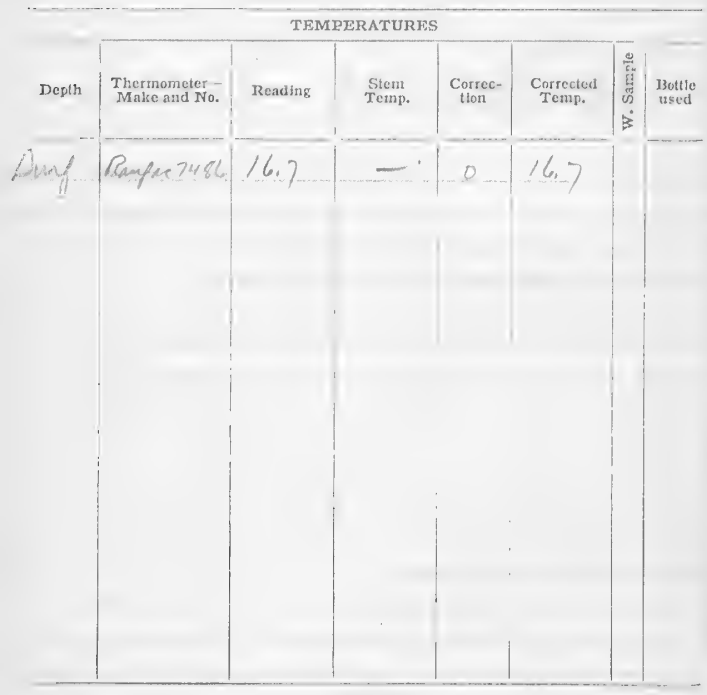

CURRENTS

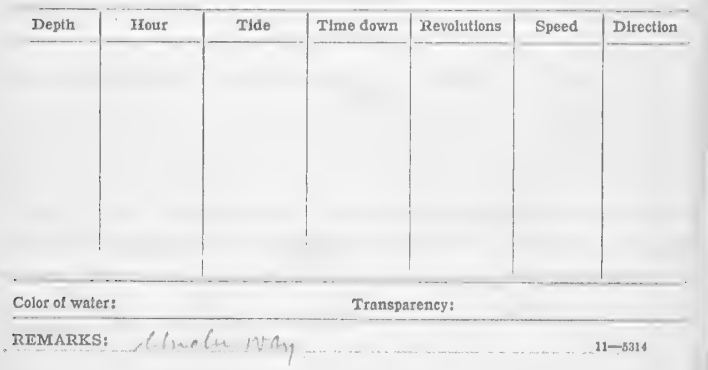


Hour: $9: 30 \%$ M. to . M.

Position: Lat. $31^{\circ}-04-00^{\prime}$ ip Long. $117-39-30^{\circ} \pm$

Locality:

Depth:

Bottom:

Sample:

Wind: Direction,

Force,

Sea:

Sky:

TEM PTRATURES

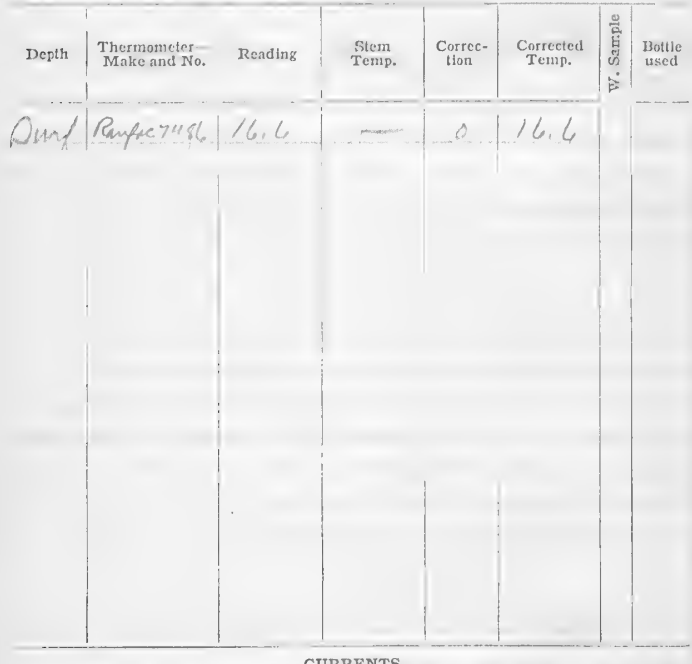

CURRENTS

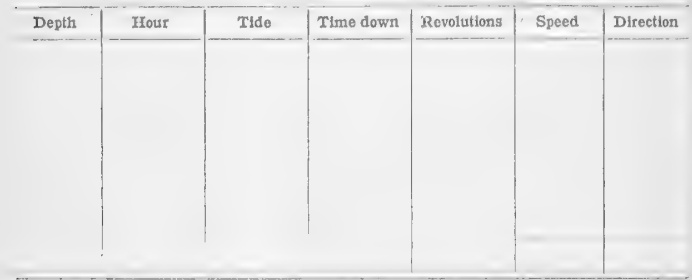


Hour: $/ O ; O D P . \mathbf{M}$. to $\ldots \ldots$. . . .

Position: Lat. $31^{\circ}-00^{\prime}-30^{\prime \prime} \geqslant$ Long. $117-40-301,1$

\section{Locality:}

Depth:

Bottom:

Sample:

Wind: Direction,

Sea:

-

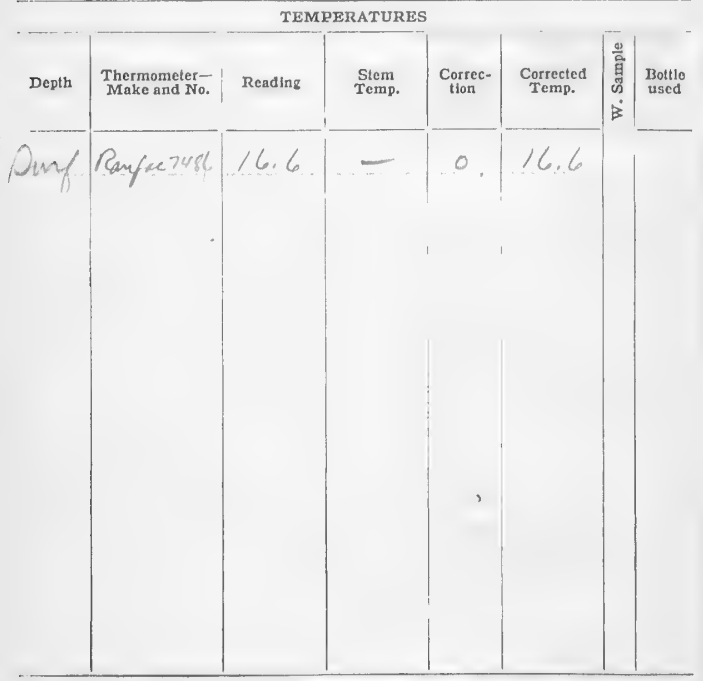

CURRENTS

\begin{tabular}{l|c|c|c|c|c|c}
\hline Depth & Houx & Tide & Time down & Revolutions & Speed & Direction \\
\hline & & & & & \\
& & & & & \\
& & & & & & \\
\hline
\end{tabular}

Color of water:

Transparency:

Force,

Sky: 


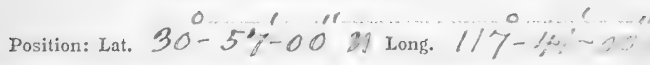

\section{Locality:}

Depth:

Bottom:

Sample:

Wind: Direction,

Force,

\section{Sea:}

Sky:

TEMPERATURES

\begin{tabular}{|c|c|c|c|c|c|c|c|}
\hline Depth & $\begin{array}{l}\text { Thermometer } \\
\text { Mako and No. }\end{array}$ & Reading & $\begin{array}{l}\text { Stcm } \\
\text { Temp. }\end{array}$ & $\begin{array}{c}\text { Correc- } \\
\text { tion }\end{array}$ & $\begin{array}{l}\text { Corrected } \\
\text { Temp. }\end{array}$ & 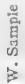 & $\begin{array}{l}\text { Botlic } \\
\text { used }\end{array}$ \\
\hline
\end{tabular}

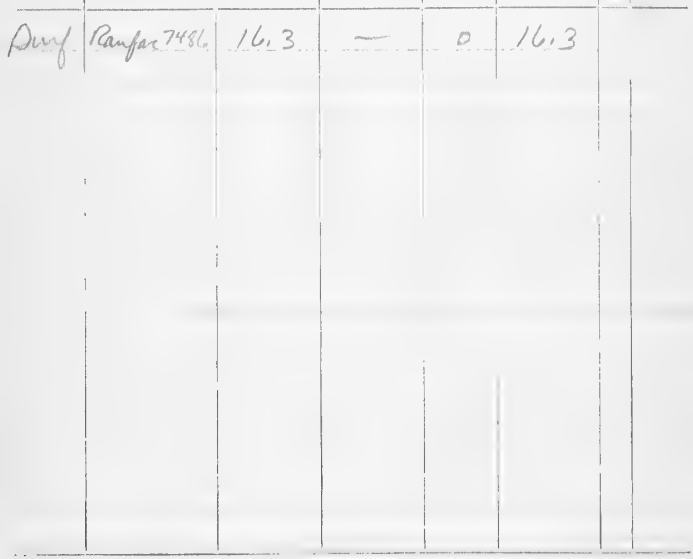

CURRENTS

\begin{tabular}{|c|c|c|c|c|c|c|}
\hline Depth & Hour & Tide & Time down & Revolutions & Speed & Direction \\
\hline & & & & & & \\
\hline & & & & & & \\
\hline & & & & & & \\
\hline & & & & & & \\
\hline & & & & & & \\
\hline & & & & & & \\
\hline
\end{tabular}

Color of water:

Transparency:

REMARKS: Llintu NMy 11- 3314 


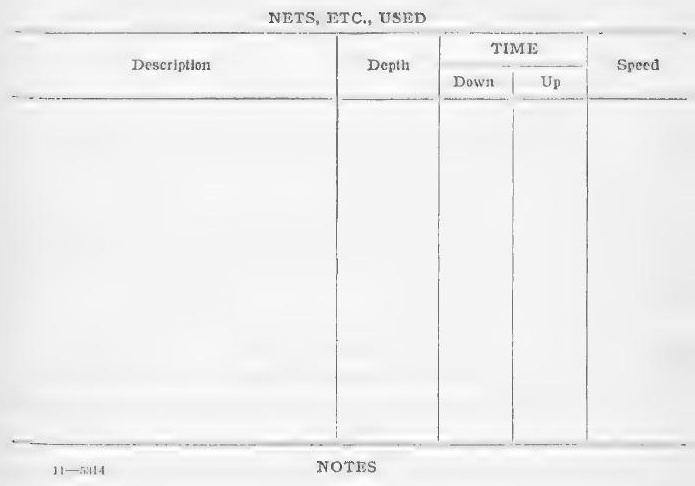

Chace of $1000-6=$ ? 
Station No. $/ 1 \%: 2 \%$

Date: . $\because 1$,

. M.

Hour: $11: 01)$ P.M.to -
191$$
\text { . }
$$

Position: Lat. $30^{\circ}-53-00^{\prime \prime} 2$ Long. $117^{0}-43^{\prime}-00$

\section{Locality:}

Depth:

Bottom:

Sample:

Wind: Direction,

Force,

Sea:

Sky:

TEMPERATURES

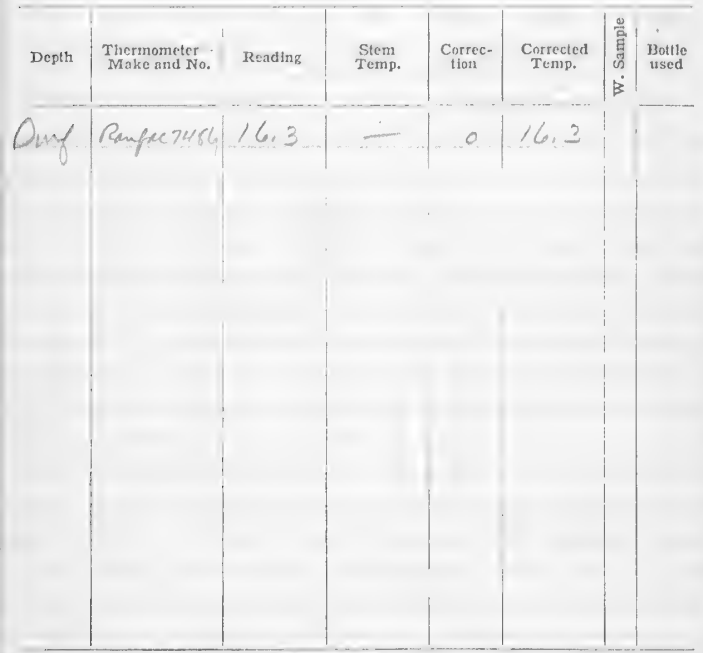

CURRENTS

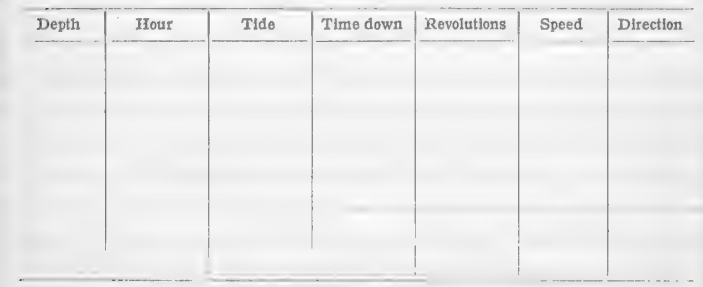

Color of water:

Transparency:

REMARKS:

Andes ivan

$11-5311$ 
NETS, ITC, USED

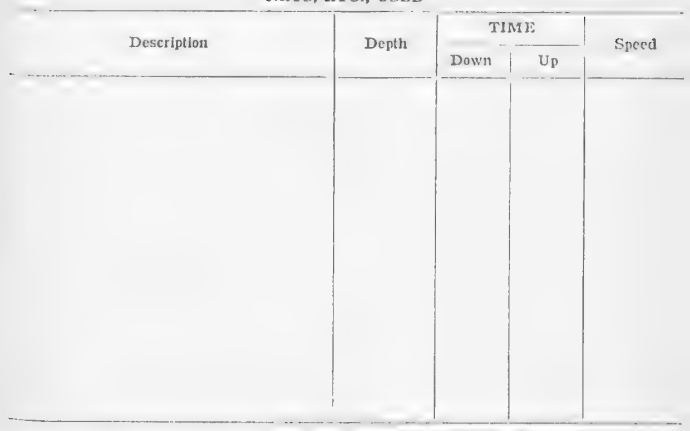

$11-2111$ NOTES

$i=1, \quad \cdot 14,7+i$

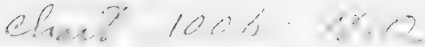




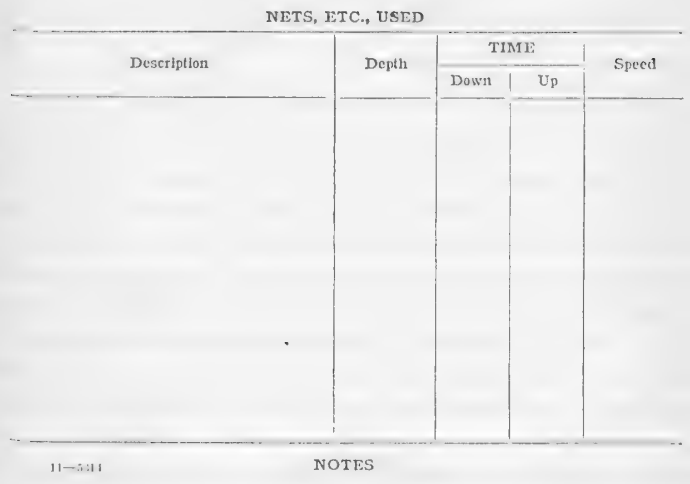

$$
\text { chen } 1008 \text { क X }
$$


Hour: $12:, \quad, \quad$ M. to . M.

Position: Lat. $30^{\circ}-4.5-00^{\prime \prime} 20$ Long. $117^{\circ}-41.00^{\prime} 1 . ;$ Locality:

Depth:

\section{Bottom:}

Sample:

Wind: Direction,

Sea:
Force,

Sky:

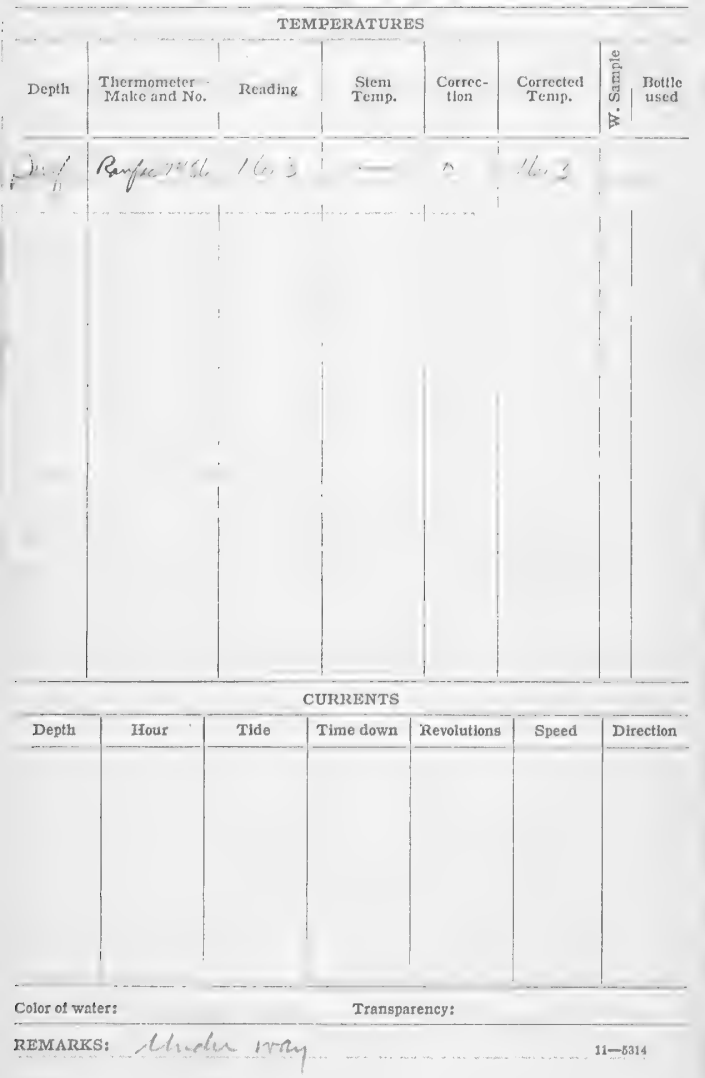




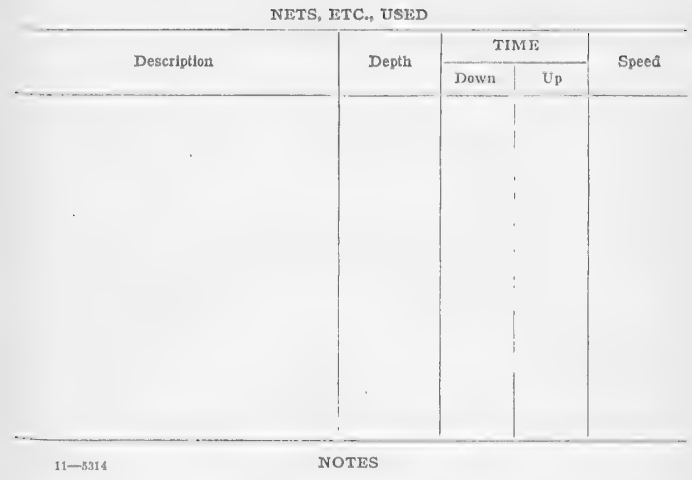

Psy. W/4.4D/5.8

chaid $1006-18.0$. 
Hour: $12: 30$ A.M. to . . . M.

Position: Lat. $30^{\circ}-41-00^{\prime \prime} 7$ Long. $^{\prime} 117-4-5^{\prime} 00^{\prime \prime}$ Li)

Locality:

Depth: _....... Bottom: . Sample:

Wind: Direction,

Sea:
Force,

Sky:

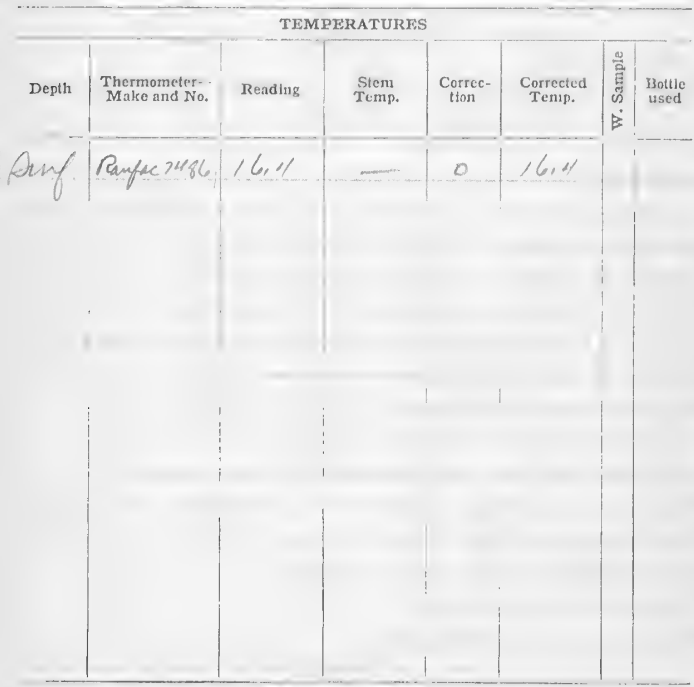

\section{CURRENTS}

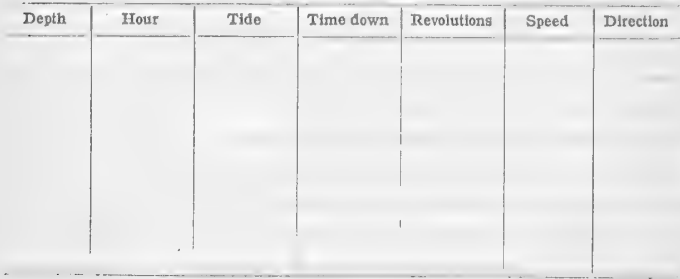


NITS, ETC., USED

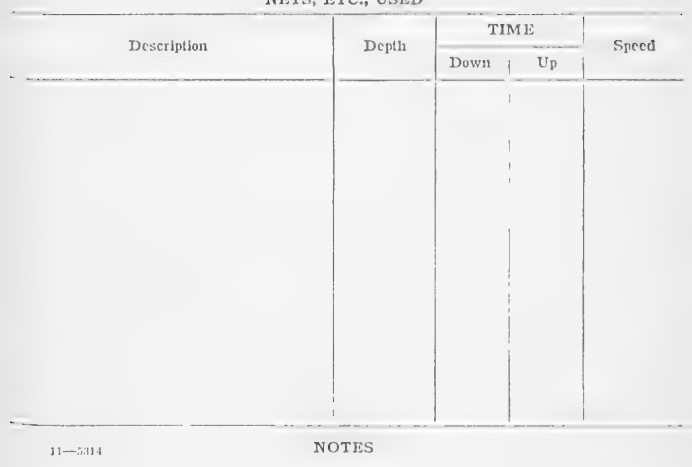

Cix-? 1:16-y+2 
Hour: $/$ io 0 A.M. to -

. M.

Position: Lat. $30^{\circ}-37^{\prime}-30^{\prime \prime}$ is Long. $1 / 7-146^{\prime}-00^{\prime \prime}$

Locality:

Depth:

Bottom:

Sample:

Wind: Direction,

Sea:
Force,

Sky:

TEMPERATURES

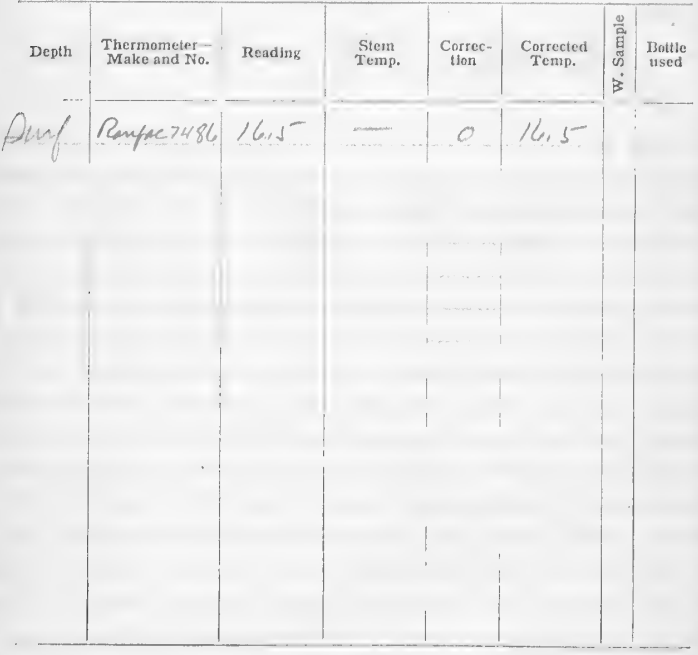

CURRENTS

\begin{tabular}{l|l|l|l|l|l|l|l|}
\hline Depth & Tide & Time down & Revolutions & Speed & Direction \\
\hline & & & & & \\
& & & & & \\
& & & &
\end{tabular}

Color of water:

Transparency:

REMARKS:

Lhole may

$11-8314$ 
NETS, ETC., USED

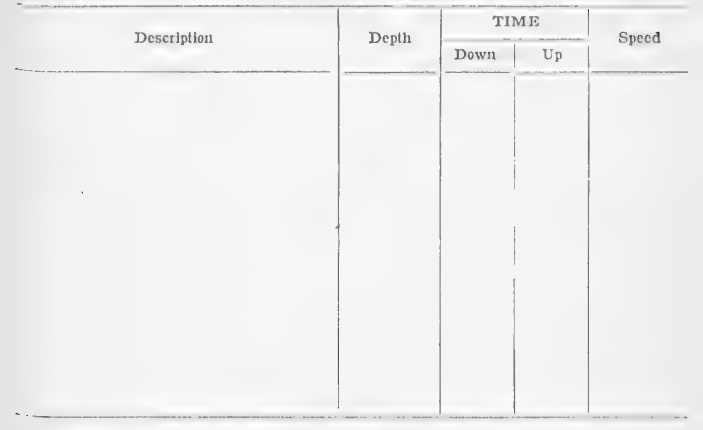

$1-i, \quad \quad \quad 14,510,1$,

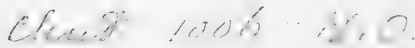


Hour: $1: 30$ A.M. to . M.

Position: Lat. $30^{\circ}-34^{\prime}-00^{\prime \prime} \geq$ Long. $117-47-00^{\circ}$

\section{Locality:}

Depth:

Bottom:

Sample:

Wind: Direction,

Force,

Sea:

Sky:

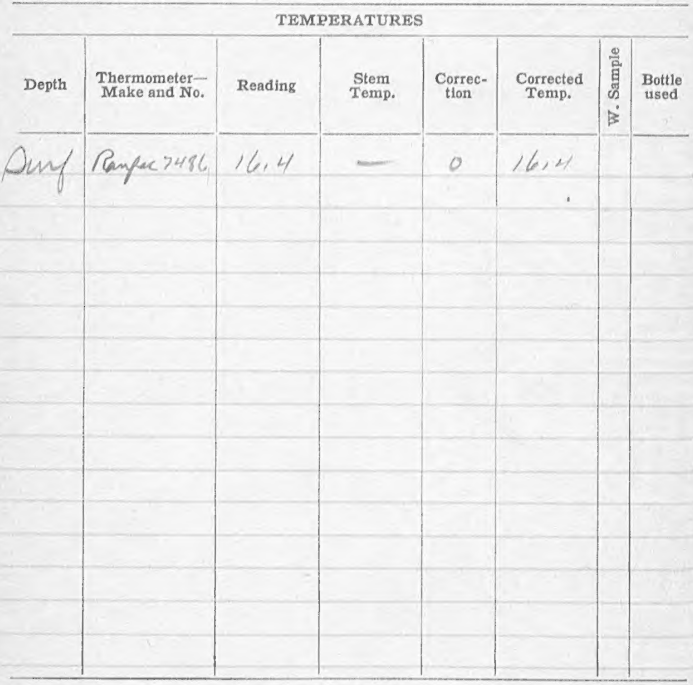

CURRENTS

\begin{tabular}{l|l|l|l|l|l|l}
\hline Depth & Hour & Tide & Time down & Revolutions & Speed & Direction \\
\hline & & & & & \\
\hline & & & & & & \\
& & & & & & \\
& & & & & & \\
\hline
\end{tabular}

Transparency:

REMARKS: Chualu rray 
NETS, ETC., USED

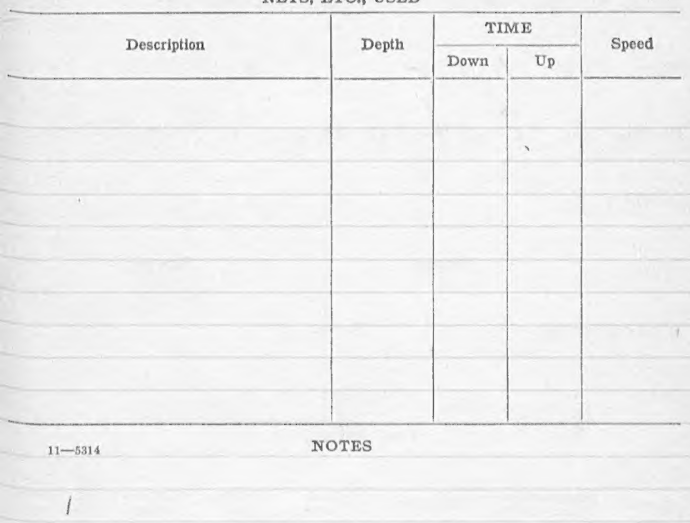

$$
\text { chant } 1006-18.0 \text {. }
$$


Station No. 16842

Date:

ary 18

1916

Hour: 2:00 A.M. to -

. M.

Position: Lat. $30^{\circ}-29^{\circ}-30^{\prime \prime} \times$ Long. $117^{\circ}-48^{\prime}-00^{\prime \prime}$

\section{Locality:}

Depth:

Bottom:

Sample:

Wind: Direction

Force,

Sea:

Sky:

TEMPERATURES

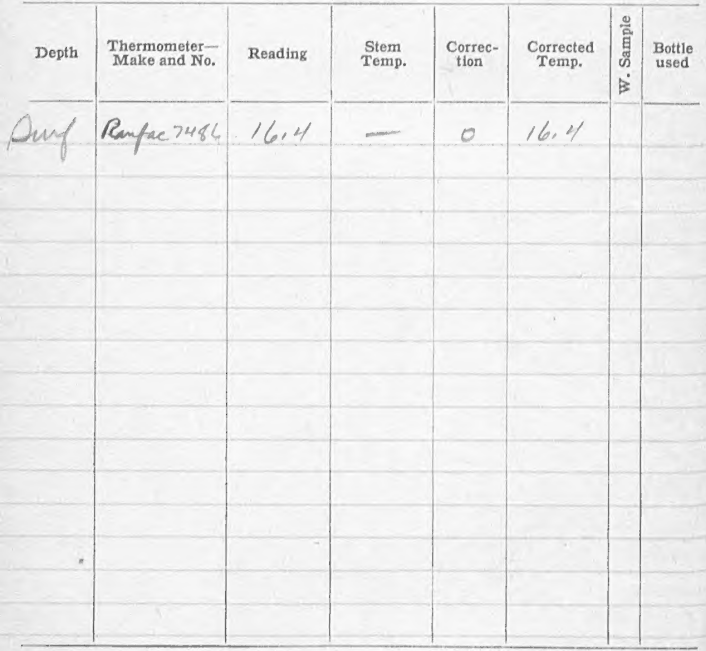

CURRENTS

\begin{tabular}{l|c|c|c|c|c|c}
\hline Depth & Hour & Tide & Time down & Revolutions & Speed & Direction \\
\hline & & & & & & \\
\hline & & & & & & \\
& & & & & & \\
& & & & & & \\
\hline
\end{tabular}

REMARKs: limelu $17 \mathrm{my}$ 
NETS, ETC., USED

\begin{tabular}{l|l|l|l|l|l}
\hline Description & Depth & \multicolumn{2}{|c|}{ TIME } & \multirow{2}{*}{ Speed } \\
\hline & & & & & \\
\hline & & & & \\
\hline & & & & \\
\hline
\end{tabular}

$11-5314$

NOTES

Psy. W 14,0 D 15,7

chand $1006-\% .0$.

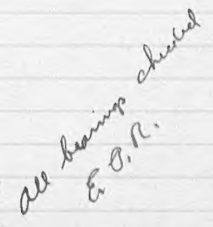

